\title{
Feedback stability of negative imaginary systems
}

DOI:

10.1109/TAC.2017.2688579

\section{Document Version}

Accepted author manuscript

Link to publication record in Manchester Research Explorer

\section{Citation for published version (APA):}

Lanzon, A., \& Chen, H-J. (2017). Feedback stability of negative imaginary systems. IEEE Transactions on

Automatic Control, 62(11), 5620 - 5633. https://doi.org/10.1109/TAC.2017.2688579

\section{Published in:}

IEEE Transactions on Automatic Control

\section{Citing this paper}

Please note that where the full-text provided on Manchester Research Explorer is the Author Accepted Manuscript or Proof version this may differ from the final Published version. If citing, it is advised that you check and use the publisher's definitive version.

\section{General rights}

Copyright and moral rights for the publications made accessible in the Research Explorer are retained by the authors and/or other copyright owners and it is a condition of accessing publications that users recognise and abide by the legal requirements associated with these rights.

\section{Takedown policy}

If you believe that this document breaches copyright please refer to the University of Manchester's Takedown Procedures [http://man.ac.uk/04Y6Bo] or contact uml.scholarlycommunications@manchester.ac.uk providing relevant details, so we can investigate your claim.

\section{OPEN ACCESS}




\title{
Feedback stability of negative imaginary systems
}

\author{
Alexander Lanzon, Senior Member, IEEE and Hsueh-Ju Chen
}

\begin{abstract}
This paper extends the robust feedback stability theorem of negative imaginary systems by removing restrictive assumptions on the instantaneous gains of the systems that were imposed in the earlier literature, and it further generalises this robust analysis result into the case that allows negative imaginary systems to have poles at the origin. In so doing, we extend the class of negative imaginary systems for which this robust stability theorem is applicable. We also show that this new generalised necessary and sufficient result specialises to the earlier theorems under the same assumptions. We additionally prove that the previously known de gain condition is not only necessary and sufficient for robust feedback stability under the earlier specified instantaneous gain assumptions, but is also necessary and sufficient for robust feedback stability under new, different and equally simple assumptions. The general robust feedback stability theorem for negative imaginary systems with free body dynamics (i.e. poles at the origin) derived in this paper also specialises to the case that is only applicable for the negative imaginary system without poles at the origin. Since the results for negative imaginary systems with free body dynamics developed in this paper depend on the existence of a matrix $\Psi$ with certain properties, we also propose a systematic construction of this matrix $\Psi$ and show that construction of one such $\Psi$ is sufficient for determining the feedback stability of the closed-loop system. Finally, examples are used to demonstrate the applicability of the results.
\end{abstract}

Index Terms-Negative imaginary systems, robust control, feedback stability.

\section{INTRODUCTION}

$\mathbf{N}$ EGATIVE imaginary systems theory was introduced in [1]. In a single-input single-output (SISO) frequency domain setting, the negative imaginary notion broadly means that the positive frequency branch of the Nyquist plot has negative imaginary part. In a SISO time domain setting, the negative imaginary notion broadly means that the output of a system follows but lags behind any sinusoidal input to the system by not more than $180^{\circ}$. In contrast to positive realness, the definitions of negative imaginary systems impose weaker restrictions on the relative degree of the transfer function and do not exclude all unstable zeros [2]. The original development of negative imaginary theory was motivated by inertial systems, all of which can be described by Newton's second law of motion, whose actuation is provided by a force or torque input and whose sensing is obtained through a colocated position or angle output measurement [1, 3]. Negative imaginary systems theory was found to be suitable for a wide variety of applications including nano-positioning control due to piezoelectric transducers and capacitive sensors (e.g. [4-6]) and in multi-agent networked systems (e.g. [7-9]).

Alexander Lanzon and Hsueh-Ju Chen are with the School of Electrical and Electronic Engineering, University of Manchester, Manchester M13 9PL, U.K. (E-mail addresses: Alexander.Lanzon@manchester.ac.uk; hsuehju.chen@manchester.ac.uk).

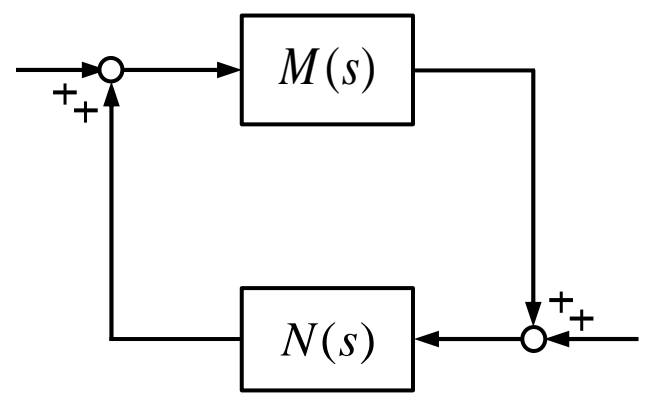

Fig. 1. Positive feedback interconnection.

The notion of negative imaginary systems also specialises to the subclass of lossless systems [10].

In view of the prevalence of negative imaginary systems in different domains, robust stability of the positive feedback interconnection of a negative imaginary system $M(s)$ and a strictly negative imaginary systems $N(s)$ was investigated in [1]. Under the assumptions that the instantaneous gain of the strictly negative imaginary system $N(s)$ is positive semidefinite, i.e. $N(\infty) \geq 0$, and the open loop transfer function is strictly proper, i.e. $M(\infty) N(\infty)=0$, [1] showed that the feedback interconnection in Fig. 1 denoted by $[M(s), N(s)]$ is internally stable if and only if the dc loop gain condition $\bar{\lambda}[M(0) N(0)]<1$ is satisfied, where $\bar{\lambda}[\cdot]$ denotes the maximum eigenvalue for a matrix with only real eigenvalues. Note that $\bar{\lambda}[\cdot]<1$ is a one-sided restriction, as $\bar{\lambda}[\cdot]$ can be either positive or negative. This result was also found to hold true in [11] when the definition of a negative imaginary system was extended to allow for poles on the imaginary axis but not at the origin. Subsequent modifications in [12] also allow for poles at the origin (under some restrictive assumptions) but this relaxation makes the result significantly more complicated and hence less transparent. These stability conditions are robust in the sense that the internal stability conclusion is invariant to negative imaginary perturbations provided that the aforementioned $\mathrm{dc}$ and instantaneous gain conditions are preserved (see Theorem 6 in [2] for negative imaginary perturbations that preserve the class).

Despite many developments in negative imaginary systems theory including for example extensions or connections to infinite dimensional systems [13], Hamiltonian systems [14], descriptor systems [15], non-rational systems [2, 16], discretetime systems [17], finite frequency negative imaginary systems [18], mixtures of small-gain and negative imaginary properties [19] and negative imaginary control synthesis [2022], the aforementioned robust feedback stability theorem has remained unchanged since [1] and hence underpins the motivation and application of all this work. When the assumptions of the feedback stability theorem in [1] and [11] do 
not hold, such as $N(\infty)$ may be sign-indefinite, the dc loop gain $\bar{\lambda}[M(0) N(0)]<1$ may not be an appropriate necessary and sufficient test for internal stability of the feedback interconnection. Very recently [23], has attempted to relax these assumptions for negative imaginary systems without poles at the origin by invoking Integral Quadratic Constraint (IQC) theory. A pair of complementary IQCs were introduced at dc and infinite frequency to attempt to relax the aforementioned assumptions. However, the methods of [23] only yield a conservative sufficient condition as will be illustrated in the numerical example section. Unlike the sufficient conditions of [23], this paper directly derives necessary and sufficient conditions by building on the work of $[1,11]$ and we handle the complete class of negative imaginary systems with or without poles at the origin and on the imaginary axis. In so doing, we demonstrate that the mechanism to remove these assumptions is not IQC theory. As indicated above, the conditions in [12] are derived for negative imaginary systems that allow for poles on the imaginary axis and at the origin. However, the conditions obtained in [12] are highly technical, require computation of several matrix factorisations which make the results less intuitive than earlier work and impose a number of additional assumptions for the results to hold amongst which is the restriction that the negative imaginary system has to be strictly proper. This paper also generalises the work in [12] by relaxing the aforementioned assumptions and provides simple necessary and sufficient conditions that invoke directly the steady-state and instantaneous gains of the systems, thereby avoiding cumbersome matrix factorisations and hence making the results significantly more intuitive.

The main contributions of this work are thus: (i) removal of the restrictive assumptions in the robust analysis theorem that underpins all of negative imaginary systems theory; (ii) derivation of new necessary and sufficient conditions that reduce to the earlier necessary and sufficient conditions under the same assumptions imposed in literature; (iii) derivation of a new set of simple and easy-to-check assumptions under which the intuitive dc gain condition is both necessary and sufficient; (iv) specialisation of the results in a SISO setting which illustrates a number of tests not easily explained in a multiple-input multiple-output (MIMO) setting; (v) demonstration that in certain scenarios a mixture of dc and infinite frequency gains are essential to characterise the necessary and sufficient conditions for robust feedback stability (this is counter intuitive); (vi) derivation of generalised necessary and sufficient robust feedback stability conditions that can be applied to negative imaginary systems having poles at the origin; (vii) demonstration that the matrix $\Psi$, satisfying certain properties, used in the two main stability theorems always exists and we provide a systematic construction for this matrix $\Psi$; (viii) demonstration that the conditions which involve the matrix $\Psi$ are either satisfied for the entire set of matrices $\Psi$ that fulfil certain properties or violated for the entire same set of matrices $\Psi$. This means one only needs to check the conditions on one $\Psi$ to determine the conclusion (i.e. there is no need for searching across the set of possible matrices $\Psi$ ); (ix) unification of all robust feedback stability results that appeared in all earlier literature to date into one general theory; and (x) illustration via two numerical examples of either the inapplicability or conservativeness of earlier results and correspondingly the usefulness of the derived general theory.

Notation: $\mathscr{R} \mathscr{H}_{\infty}$ denotes the set of real, rational, stable transfer function matrices. $\mathbb{R}^{m \times n}$ and $\mathbb{C}^{m \times n}$ denote real and complex matrices that have $n$ columns and $m$ rows respectively. $\lambda_{i}(A)$ denotes the $i$ th eigenvalue of a square complex matrix $A . \bar{\lambda}(A)$ and $\underline{\lambda}(A)$ denote the largest and smallest eigenvalue of a square complex matrix $A$ that has only real eigenvalues. $A^{*}$ and $A^{T}$ denote the complex conjugate transpose and transpose of a complex matrix $A$ respectively. $A^{-*}$ and $G^{\sim}(s)$ are shorthand for $\left(A^{-1}\right)^{*}$ and $G(-s)^{T}$ respectively. $\mathfrak{R}(a)$ denotes the real part of a complex number $a$. Let $\operatorname{ker}(A)$ denote the kernel of matrix $A$. A square transfer function matrix $G(s)$ is said to have full normal rank if $\exists s \in \mathbb{C}: \operatorname{det}(G(s)) \neq 0$.

\section{Preliminary Lemmas}

First, let us recall the definitions of negative imaginary systems in Definitions 1 and 2.

Definition 1 ([12]): Let $R: \mathbb{C} \longrightarrow \mathbb{C}^{m \times m}$ be a real, rational, proper transfer function. Then $R(s)$ is said to be Negative Imaginary (NI) if

1) $R(s)$ has no poles in $\mathfrak{R}(s)>0$;

2) $j\left[R(j \omega)-R(j \omega)^{*}\right] \geq 0$ for all $\omega \in(0, \infty)$ except the values of $\omega$ where $j \omega$ is a pole of $R(s)$;

3) if $j \omega_{0}$ with $\omega_{0}>0$ is a pole of $R(s)$, then it is at most a simple pole and the residue matrix $K_{0}=$ $\lim _{s \rightarrow j \omega_{0}}\left(s-j \omega_{0}\right) j R(s)$ is Hermitian and positive semidefinite;

4) if $s=0$ is a pole of $R(s)$, then $\lim _{s \rightarrow 0} s^{k} R(s)=0$ for all integer $k \geq 3$ and $\lim _{s \rightarrow 0} s^{2} R(s)$ is Hermitian and positive semidefinite.

Definition 2 ([1]): Let $R: \mathbb{C} \longrightarrow \mathbb{C}^{m \times m}$ be a real, rational, proper transfer function. Then $R(s)$ is said to be Strictly Negative Imaginary (SNI) if

1) $R(s)$ has no poles in $\mathfrak{R}(s) \geq 0$;

2) $j\left[R(j \omega)-R(j \omega)^{*}\right]>0$ for all $\omega \in(0, \infty)$.

For SISO systems, the negative imaginary property ensures that the positive branch of the Nyquist plot lies below the real axis. The definitions of negative imaginary systems in $[1,11,12]$ do not require the negative imaginary system to be symmetric, i.e. $G(s)=G(s)^{T}$. It is stated in [16] that there are no examples in the literature that are negative imaginary but not symmetric. We here give one such non-symmetric example.

Example 1: Let $M(s)=\left[\begin{array}{cc}\frac{-s}{s+5} & \frac{-5}{s+5} \\ \frac{-(4 s+5)}{s^{2}+6 s+5} & \frac{-s^{2}+s+15}{s^{2}+6 s+5}\end{array}\right]$ and $\left[\begin{array}{l|l}A & B \\ \hline C & D\end{array}\right]$ be a minimal realization of $M(s)$ where $A=$ $\left[\begin{array}{cc}0 & 1 \\ -5 & -6\end{array}\right], B=\left[\begin{array}{cc}-1 & -2 \\ 6 & -3\end{array}\right], C=\left[\begin{array}{cc}1 & 1 \\ -2 & -1\end{array}\right]$, and $D=$ $\left[\begin{array}{cc}-1 & 0 \\ 0 & -1\end{array}\right]$. We demonstrate that $M(s)$ is a strictly negative 
imaginary system despite it not being symmetric. To do this, we use the negative imaginary lemma in $[1,11]$ and also check for transmission zeros on the imaginary axis. $M(s)$ is negative imaginary because there exists a real matrix $Y=$ $\left[\begin{array}{cc}3 & -3 \\ -3 & 4\end{array}\right]>0$ such that $A Y+Y A^{*}=\left[\begin{array}{cc}-6 & 7 \\ 7 & -18\end{array}\right] \leq 0$ and $B=-A Y C^{*}$. Furthermore, $\operatorname{det}\left(M(s)-M^{\sim}(s)\right)=$ $\frac{59 s^{2}}{s^{4}-26 s^{2}+25} \neq 0 \quad \forall s \in\{s=j \omega: \omega \in(0, \infty)\}$. Hence, $M(s)$ is negative imaginary and there is no transmission zeros of $M(s)-M^{\sim}(s)$ when $s=j \omega \forall \omega \in(0, \infty)$ which implies that $M(s)$ must be strictly negative imaginary.

We now give a few technical lemmas that will be used to prove the main results. We start by giving alternative representations for $\lambda_{i}\left[(I-A B)^{-1}(C D-I)\right]$.

Lemma 3: Suppose that $A, B, C$ and $D$ are real symmetric matrices and $\operatorname{det}(I-A B) \neq 0$. Then,

$$
\begin{aligned}
\lambda_{i}\left[(I-A B)^{-1}(C D-I)\right] & =\lambda_{i}\left[(C D-I)(I-A B)^{-1}\right] \\
& =\lambda_{i}\left[(I-B A)^{-1}(D C-I)\right] \\
& =\lambda_{i}\left[(D C-I)(I-B A)^{-1}\right] .
\end{aligned}
$$

Proof: The first equality is via a similarity transform with $(I-A B)$. The second equality is via $\lambda_{i}(X)=\lambda_{i}\left(X^{T}\right)$. The third equality is via a similarity transform with $(I-B A)$.

Next we show that certain matrix structures have real eigenvalues.

Lemma 4: Suppose that $A, B$ and $C$ are real symmetric matrices satisfying $C \geq B$ and $\operatorname{det}(I-A B) \neq 0$. Then,

$$
\lambda_{i}\left[(I-A B)^{-1}(A C-I)\right] \in \mathbb{R} \quad \forall i .
$$

Proof: Trivial via rearrangement into a symmetric matrix.

$$
\begin{aligned}
& \lambda_{i}\left[(I-A B)^{-1}(A C-I)\right] \\
& \quad=\lambda_{i}\left[(I-A B)^{-1} A(C-B)\right]-1 \\
& \quad=\lambda_{i}\left[(C-B)^{1 / 2}(I-A B)^{-1} A(C-B)^{1 / 2}\right]-1 .
\end{aligned}
$$

The next lemma manipulates scalar inequalities. This will be useful in deriving the main results of Section IV.

Lemma 5: Given four scalars $a, b, \alpha$, and $\beta$ with $\alpha \geq 0$ and $\beta \geq 0$. If $a b<1$ and $(a+\alpha)(b+\beta)<1$, then $(a+\alpha) b<1$.

Proof: If $b \leq 0$, then $(a+\alpha) b=a b+\alpha b \leq a b<1$. If on the other hand $b>0$, then $b+\beta>0$ and hence $(a+\alpha) b=$ $(a+\alpha)(b+\beta) \frac{b}{(b+\beta)}<1$.

We now show that $s=j \omega_{0}$ with $\omega_{0} \in(0, \infty)$ is not a transmission zero of $I-M(s) N(s)$ when $M(s)$ and $N(s)$ are negative imaginary systems and $s=j \omega_{0}$ is a pole of $M(s)$.

Lemma 6: Let $M(s)$ be a negative imaginary system and $N(s)$ be a strictly negative imaginary system. Assume $s=$ $j \omega_{0}$ with $\omega_{0} \in(0, \infty)$ is a simple pole of $M(s)$. Then $I-$ $M(s) N(s)$ has no transmission zero at $s=j \omega_{0}$.

Proof: Since $s=j \omega_{0}$ with $\omega_{0} \in(0, \infty)$ is a simple pole of $M(s), M(s)$ can be factored as $M(s)=M_{1}(s)+\frac{-j A}{s-j \omega_{0}}$ where $A=A^{*} \geq 0$ and $M_{1}(s)$ is analytic in the neighborhood of $s=j \omega_{0}$.

Let $0 \neq x \in \operatorname{ker}(A)$ and choose a sufficiently small $\delta>0$. Since $M(s)$ is a negative imaginary system,

$$
j\left[M(j \omega)-M(j \omega)^{*}\right] \geq 0 \quad \forall \omega \in\left\{0<\left|\omega-\omega_{0}\right|<\delta\right\}
$$

$$
\begin{aligned}
\Leftrightarrow & {[j M(j \omega)]+[j M(j \omega)]^{*} \geq 0 \quad \forall \omega \in\left\{0<\left|\omega-\omega_{0}\right|<\delta\right\} } \\
\Rightarrow & x^{*}\left[[j M(j \omega)]+[j M(j \omega)]^{*}\right] x \geq 0 \quad \forall \omega \in\left\{0<\left|\omega-\omega_{0}\right|\right. \\
& <\delta\} \\
\Leftrightarrow & x^{*}\left[\left[j M_{1}(j \omega)\right]+\left[j M_{1}(j \omega)\right]^{*}\right] x \geq 0 \quad \forall \omega \in\left\{0<\left|\omega-\omega_{0}\right|\right. \\
& <\delta\} \\
\Rightarrow & x^{*}\left[\left[j M_{1}\left(j \omega_{0}\right)\right]+\left[j M_{1}\left(j \omega_{0}\right)\right]^{*}\right] x \geq 0 \text { (by continuity of } \\
& \text { real rational functions and because } M_{1}(s) \text { is analytic near } \\
& \left.s=j \omega_{0}\right) .
\end{aligned}
$$

Also, since $N(s)$ is a strictly negative imaginary system,

$$
\begin{aligned}
& j\left[N(j \omega)-N(j \omega)^{*}\right]>0 \quad \forall \omega \in(0, \infty) \\
\Leftrightarrow & {[j N(j \omega)]+[j N(j \omega)]^{*}>0 \quad \forall \omega \in(0, \infty) } \\
\Leftrightarrow & {[j N(j \omega)]^{-1}+[j N(j \omega)]^{-*}>0 \quad \forall \omega \in(0, \infty) } \\
& (\text { since } N(j \omega) \text { is nonsingular } \forall \omega \in(0, \infty) \text { via [1]) } \\
\Rightarrow & {\left[j N\left(j \omega_{0}\right)\right]^{-1}+\left[j N\left(j \omega_{0}\right)\right]^{-*}>0 } \\
\Rightarrow & x^{*}\left[\left[j N\left(j \omega_{0}\right)\right]^{-1}+\left[j N\left(j \omega_{0}\right)\right]^{-*}\right] x>0 .
\end{aligned}
$$

From the above two conditions, we now have

$$
\begin{aligned}
& x^{*}\left[\left[j N\left(j \omega_{0}\right)\right]^{-1}+\left[j M_{1}\left(j \omega_{0}\right)\right]\right] x \\
& \quad+x^{*}\left[\left[j N\left(j \omega_{0}\right)\right]^{-1}+\left[j M_{1}\left(j \omega_{0}\right)\right]\right]^{*} x>0 .
\end{aligned}
$$

However, we need to show that $I-M(s) N(s)$ has no transmission zero at $s=j \omega_{0}$, which is equivalent to $[j N(s)]^{-1}+$ $[j M(s)]$ has no transmission zero at $s=j \omega_{0}$.

We show this via contradiction. Suppose $s=j \omega_{0}$ with $\omega_{0}>0$ is a transmission zero of $[j N(s)]^{-1}+[j M(s)]$. Then $\exists y \in \mathbb{C}^{m}$ with $y \neq 0$ such that $\left[[j N(s)]^{-1}+[j M(s)]\right] y=0$ at $s=j \omega_{0}$. Expanding the above equation, we have that the given $y$ must satisfy $[j N(s)]^{-1} y+\left[j M_{1}(s)\right] y=\frac{-A y}{s-j \omega_{0}}$. But in the limit as $s \rightarrow j \omega_{0}$, the left-hand side is finite and the righthand side is infinite when $y \notin \operatorname{ker}(A)$. Hence $y \in \operatorname{ker}(A)$. Then $\left[[j N(s)]^{-1}+[j M(s)]\right] y=0$ at $s=j \omega_{0}$ implies $\left[j N\left(j \omega_{0}\right)\right]^{-1} y+\left[j M_{1}\left(j \omega_{0}\right)\right] y=0$ which in turn implies that (1) cannot be fulfilled for the choice $x=y$ as $x$ was an arbitrary non-zero vector in $\operatorname{ker}(A)$. Then, by contradiction, $I-M(s) N(s)$ has no transmission zero at $s=j \omega_{0}$.

The following lemma provides some simple manipulations of negative imaginary systems.

Lemma 7: Let $M(s)$ be a square, real, rational and proper transfer function matrix. Then

1) $M(s)$ is negative imaginary if and only if $M(s)-M(\infty)$ is negative imaginary and $M(\infty)=M(\infty)^{T}$;

2) $M(s)-M(\infty)$ is negative imaginary if and only if $s[M(s)-M(\infty)]$ is positive real.

Proof: 1) Trivial. 2) See [24, Lemma 2].

The next lemma extends [1, Lemma 2] to negative imaginary systems that can possibly have poles on the $j \omega$-axis excluding the origin.

Lemma 8: Let $M(s)$ be a negative imaginary system without poles at the origin. Then $M(0)=M(0)^{T}, M(\infty)=M(\infty)^{T}$ and $M(0) \geq M(\infty)$.

Proof: The results are trivial when $M(s)$ is static. When $M(s)$ is dynamic, let it have a minimal realisation $\left[\begin{array}{l|l}A & B \\ \hline C & D\end{array}\right]$. Using [11, Lemma 7], we get $D=D^{T}$ and $M(0)-M(\infty)=$ $-C A^{-1} B=C A^{-1} A Y C^{*}=C Y C^{*} \geq 0$. The results then follow. 


\section{Main Results: Part 1- No Poles AT The ORIGiN}

References $[1,11]$ derive a necessary and sufficient condition for internal stability of the positive feedback interconnection of a negative imaginary system without poles at the origin and a strictly negative imaginary system as shown in Fig. 1 under two assumptions at infinite frequency. Here, we consider the same feedback structure but remove these two assumptions imposed in $[1,11]$, hence obtaining a generalized stability condition. While the condition in $[1,11]$ for determining internal stability of the closed-loop system requires a test only on the dc loop gain, in our generalised setting, the internal stability conditions in Theorems 9 and 14 depend on both the frequencies 0 and $\infty$. Although the resulting stability conditions involving a mixture of frequencies are surprising, this is indeed correct and will be explained in detail in the next section when the results will be specialised to the SISO setting and given a Nyquist interpretation.

The matrix $I-N(0) M(\infty)$ is invertible in the third condition of Theorem 9 because the second condition (in conjunction with the first condition) in Theorem 9 guarantees its invertibility. Also, the matrices $[I-M(\infty) N(\infty)]^{-1}(M(\infty) N(0)-I)$ and $[I-N(0) M(\infty)]^{-1}(N(0) M(0)-I)$ in the conditions of Theorem 9 have only real eigenvalues via Lemmas 4 and 8 .

Theorem 9: Let $M(s)$ be a negative imaginary system without poles at the origin and $N(s)$ be a strictly negative imaginary system. Then $[M(s), N(s)]$ is internally stable if and only if

$$
\begin{aligned}
& I-M(\infty) N(\infty) \text { is nonsingular, } \\
& \bar{\lambda}\left[[I-M(\infty) N(\infty)]^{-1}(M(\infty) N(0)-I)\right]<0, \text { and } \\
& \bar{\lambda}\left[[I-N(0) M(\infty)]^{-1}(N(0) M(0)-I)\right]<0 .
\end{aligned}
$$

Proof: This proof closely follows the proof of the corresponding result given in [1] with appropriate modifications in pertinent statements to allow relaxation of the restrictive assumptions at $\infty$ frequency.

$$
\text { Let } N(s)=\left[\begin{array}{c|c}
\bar{A} & \bar{B} \\
\hline \bar{C} & \bar{D}
\end{array}\right] \text { be a minimal realisation. Then, by }
$$

the assumptions of this theorem and using [11, Lemma 8], $\bar{A}$ is Hurwitz, $\bar{D}=\bar{D}^{*}$, and there exists a real matrix $\bar{Y}=\bar{Y}^{*}>0$ such that

$$
\bar{A} \bar{Y}+\bar{Y} \bar{A}^{*} \leq 0 \text { and } \bar{B}=-\bar{A} \bar{Y} \bar{C}^{*} .
$$

The proof is brief when $M(s)$ is a static matrix $K$, i.e. $M(s)=K$, as the first condition in the theorem statement is equivalent to well-posedness, the third condition is trivially fulfilled and the second condition is equivalent to $(I-K N(s))^{-1} \in \mathcal{R} \mathcal{H}_{\infty}$ via the following argument: $(I-K N(s))^{-1} \in \mathcal{R H}_{\infty}$ is equivalent to $\hat{\mathcal{A}}=\bar{A}+$ $\bar{B}(I-K \bar{D})^{-1} K \bar{C}=\mathcal{P} \mathcal{Q}$ is Hurwitz, where $\mathcal{P}=\bar{A} \bar{Y}$ and $\mathcal{Q}=\bar{Y}^{-1}-\bar{C}^{*}(I-K \bar{D})^{-1} K \bar{C}$. Then $\hat{\mathcal{A}}$ is Hurwitz if and only if $\mathcal{Q}>0$ which is equivalent to the second condition. This concludes the proof when $M(s)$ is a static matrix.

Next, consider the case when $M(s)$ has dynamics. Let $M(s)=\left[\begin{array}{l|l}A & B \\ \hline C & D\end{array}\right]$ be a minimal realization. Then, by the assumptions of this theorem and using [11, Lemma 7], $\operatorname{det}(A) \neq$
$0, D=D^{*}$, and there exists a real matrix $Y=Y^{*}>0$ such that

$$
A Y+Y A^{*} \leq 0 \text { and } B=-A Y C^{*} .
$$

Define $U=I-D \bar{D}, \quad V=I-\bar{D} D, \Phi=\left[\begin{array}{cc}A Y & 0 \\ 0 & \bar{A} \bar{Y}\end{array}\right]$ and $T=\left[\begin{array}{cc}Y^{-1}-C^{*} \bar{D} U^{-1} C & -C^{*} V^{-1} \bar{C} \\ -\bar{C}^{*} U^{-1} C & \bar{Y}^{-1}-\bar{C}^{*} U^{-1} D \bar{C}\end{array}\right]$

Then,

$[M(s), N(s)]$ is internally stable

$\Leftrightarrow I-M(\infty) N(\infty)$ is nonsingular and

$[I-M(s) N(s)]^{-1}=$

$\left[\begin{array}{cc|c}\left(\begin{array}{cc}A & B \bar{C} \\ 0 & \bar{A}\end{array}\right)+\left(\begin{array}{c}B \bar{D} \\ \bar{B}\end{array}\right) U^{-1}\left(\begin{array}{ll}C & D \bar{C}\end{array}\right) & \begin{array}{c}B \bar{D} U^{-1} \\ \bar{B} U^{-1}\end{array} \\ \hline U^{-1} C & U^{-1} D \bar{C} & U^{-1}\end{array}\right]$

(This equivalence is via Theorem 5.7 in [25] due to the facts that $N(s)$ and $M(s)$ have no poles in the open right-half plane and at the origin, and $N(s)$ having no poles nor zeros on $j \mathbb{R} /\{0\}$. Thus, $M(s) N(s)$ has no pole-zero cancellation in the closed right-half plane)

$\Leftrightarrow I-M(\infty) N(\infty)$ is nonsingular and $\mathcal{A}=\Phi T$ is Hurwitz (as the previous realization is stabilisable and detectable)

$\Leftrightarrow I-M(\infty) N(\infty)$ is nonsingular and

$T>0$ (necessity and sufficiency are proved as in the proof of [1, Theorem 5] and the proof of [11, Theorem 1] except that Lemma 6 must be used instead of $\operatorname{det}(I-M(j \omega) N(j \omega)) \neq 0 \quad \forall \omega \in(0, \infty)$ to obtain that $I-M(j \omega) N(j \omega)$ has no transmission zeros $\forall \omega \in$ $(0, \infty))$

$\Leftrightarrow I-M(\infty) N(\infty)$ is nonsingular,

$\bar{Y}^{-1}-\bar{C}^{*} U^{-1} D \bar{C}>0$, and

$\left(Y^{-1}-C^{*} \bar{D} U^{-1} C\right)-C^{*} V^{-1} \bar{C}\left(\bar{Y}^{-1}-\bar{C}^{*} U^{-1} D\right.$

$\bar{C})^{-1} \bar{C}^{*} U^{-1} C>0$

$\Leftrightarrow I-M(\infty) N(\infty)$ is nonsingular,

$\bar{Y}^{-1}-\bar{C}^{*} U^{-1} D \bar{C}>0$, and

$Y^{-1}-C^{*} \bar{D} U^{-1} C-C^{*} V^{-1}\left(I-\bar{C} \bar{Y} \bar{C}^{*} U^{-1} D\right)^{-1} \bar{C} \bar{Y}$

$\bar{C}^{*} U^{-1} C>0$

$\Leftrightarrow I-M(\infty) N(\infty)$ is nonsingular,

$\bar{Y}^{-1}-\bar{C}^{*} U^{-1} D \bar{C}>0$, and

$Y^{-1}-C^{*} \bar{D} U^{-1} C-C^{*} V^{-1}\left(I-(N(0)-\bar{D}) U^{-1} D\right)^{-1}$

$(N(0)-\bar{D}) U^{-1} C>0$

(since $\bar{C} \bar{Y} \bar{C}^{*}=N(0)-\bar{D}$ via [1, Lemma 2])

$\Leftrightarrow I-M(\infty) N(\infty)$ is nonsingular,

$\bar{Y}^{-1}-\bar{C}^{*} U^{-1} D \bar{C}>0$, and

$Y^{-1}-C^{*} \bar{D} U^{-1} C-C^{*} V^{-1}[[V-(N(0)-\bar{D}) D]$

$\left.V^{-1}\right]^{-1}(N(0)-\bar{D}) U^{-1} C>0$

$\Leftrightarrow I-M(\infty) N(\infty)$ is nonsingular,

$\bar{Y}^{-1}-\bar{C}^{*} U^{-1} D \bar{C}>0$, and

$Y^{-1}-C^{*} \bar{D} U^{-1} C-C^{*}(I-N(0) D)^{-1}(N(0)-\bar{D})$

$U^{-1} C>0$

$\Leftrightarrow I-M(\infty) N(\infty)$ is nonsingular,

$\bar{Y}^{-1}-\bar{C}^{*} U^{-1} D \bar{C}>0$, and

$Y^{-1}-C^{*}(I-N(0) D)^{-1}[(I-N(0) D) \bar{D}+(N(0)-$

$\bar{D})] U^{-1} C>0$

$\Leftrightarrow I-M(\infty) N(\infty)$ is nonsingular, 


$$
\begin{aligned}
& \bar{Y}-1-\bar{C}^{*} U^{-1} D \bar{C}>0, \text { and } \\
& Y^{-1}-C^{*}(I-N(0) D)^{-1} N(0) C>0 \\
\Leftrightarrow & I-M(\infty) N(\infty) \text { is nonsingular, } \\
& \bar{\lambda}\left[\bar{Y}^{1 / 2} \bar{C}^{*} U^{-1} D \bar{C}^{1 / 2}\right]<1, \text { and } \\
& \bar{\lambda}\left[Y^{1 / 2} C^{*}(I-N(0) D)^{-1} N(0) C Y^{1 / 2}\right]<1 \\
\Leftrightarrow & I-M(\infty) N(\infty) \text { is nonsingular, } \\
& \bar{\lambda}\left[U^{-1} D \bar{C} \bar{Y} \bar{C}^{*}\right]<1, \text { and } \\
& \bar{\lambda}\left[(I-N(0) D)^{-1} N(0) C Y C^{*}\right]<1 \\
\Leftrightarrow & I-M(\infty) N(\infty) \text { is nonsingular, } \\
& \bar{\lambda}\left[U^{-1} D(N(0)-\bar{D})-I\right]<0, \text { and } \\
& \bar{\lambda}\left[(I-N(0) D)^{-1} N(0)(M(0)-D)-I\right]<0 \\
& \left(\text { since } \bar{C} \bar{Y} \bar{C}^{*}=N(0)-\bar{D} \text { and } C Y C^{*}=M(0)-D\right. \\
& \operatorname{via}[1, \operatorname{Lemma} 2] \text { and Lemma } 8) \\
\Leftrightarrow & I-M(\infty) N(\infty) \text { is nonsingular, } \\
& \bar{\lambda}\left[[I-M(\infty) N(\infty)]^{-1}(M(\infty) N(0)-I)\right]<0, \text { and } \\
& \bar{\lambda}\left[[I-N(0) M(\infty)]^{-1}(N(0) M(0)-I)\right]<0 .
\end{aligned}
$$

Remark 10: The feedback stability conditions in Theorem 9 involve a mixture of two frequencies, i.e. 0 and $\infty$. This appears, on the surface, to be counter intuitive as if one considers the Nyquist stability criterion, one might reasonably ponder why a mixture of two frequencies is needed given that the Nyquist plot is a pointwise-in-frequency plot of $M(j \omega) N(j \omega)$. However, note that the feedback stability condition in the Nyquist stability criterion is a winding number condition (or equivalently, an encirclements condition) which is certainly not a pointwise-in-frequency condition. The fact that for certain system classes this encirclements condition can be equivalently rewritten as simpler conditions that do not require full knowledge of the transfer functions is not trivial. It is hence legitimate that the feedback stability conditions in Theorem 9 invoke more than a single frequency. Note that in the sequel we shall also show how these conditions specialise to a single frequency condition under some additional assumptions.

The next lemma states that the eigenvalues of the product of certain matrices are always real.

Lemma 11: Let $A$ and $B$ be real symmetric matrices such that either $A$ or $B$ is sign semi-definite (i.e. $\geq 0$ or $\leq 0$ ). Then $\lambda_{i}(A B) \in \mathbb{R} \forall i$.

Proof: Trivial.

If we now impose the same two restrictive assumptions $M(\infty) N(\infty)=0$ and $N(\infty) \geq 0$ as in [1, 11], the internal stability conditions in Theorem 9 reduce to the condition in $[1,11]$.

Corollary 12: Let $M(s)$ be a negative imaginary system without poles at the origin and $N(s)$ be a strictly negative imaginary system. Let $M(\infty) N(\infty)=0$ and $N(\infty) \geq 0$. Then

$[M(s), N(s)]$ is internally stable $\Leftrightarrow \bar{\lambda}[M(0) N(0)]<1$.

Proof: This is a direct consequence of Theorem 9 because:

1) $M(\infty) N(\infty)=0 \Rightarrow I-M(\infty) N(\infty)$ is nonsingular;

2) $\bar{\lambda}\left[[I-M(\infty) N(\infty)]^{-1}(M(\infty) N(0)-I)\right]<0$

$\Leftrightarrow \bar{\lambda}[M(\infty) N(0)]<1$

$\Leftrightarrow N(0)^{1 / 2} M(\infty) N(0)^{1 / 2}<I$

(since $N(0)>N(\infty) \geq 0$ via [1, Lemma 2])

$$
\begin{aligned}
& \Leftrightarrow M(\infty)<N(0)^{-1} \\
& \text { 3) } \bar{\lambda}\left[[I-N(0) M(\infty)]^{-1}(N(0) M(0)-I)\right]<0 \\
& \Leftrightarrow \bar{\lambda}\left[\left[N(0)^{-1}-M(\infty)\right]^{-1}\left(M(0)-N(0)^{-1}\right)\right]<0 \\
& \text { (since } N(0)>N(\infty) \geq 0 \text { via [1, Lemma 2]) } \\
& \Leftrightarrow\left[N(0)^{-1}-M(\infty)\right]^{-1 / 2}\left(M(0)-N(0)^{-1}\right)\left[N(0)^{-1}\right. \\
& -M(\infty)]^{-1 / 2}<0 \\
& \text { (since } N(0)^{-1}>M(\infty) \text { via above) } \\
& \Leftrightarrow M(0)-N(0)^{-1}<0 \\
& \Leftrightarrow \bar{\lambda}[M(0) N(0)]<1 \text {. }
\end{aligned}
$$

But $\bar{\lambda}[M(0) N(0)]<1 \Leftrightarrow M(0)<N(0)^{-1} \Rightarrow M(\infty)<$ $N(0)^{-1}$ (since $M(\infty) \leq M(0)$ via Lemma 8).

This concludes the proof.

Another way of simplifying the stability conditions given in Theorem 9 is to assume that $M(s)$ is a strictly proper transfer function, and under this assumption the internal stability condition obtained here is the same as the one in the previous corollary. Note that the statement in Corollary 13 was not known in previous literature. In other words, it was not known that the dc loop gain is a necessary and sufficient condition for internal stability also under a simple assumption of a strictly proper $M(s)$. Unlike [12, Remark 1] which requires $N(0)$ to be either a positive or negative definite matrix, Corollary 13 has no restriction of the sign of $N(0)$, i.e. $N(0)$ can be either sign-indefinite or singular.

Corollary 13: Let $M(s)$ be a negative imaginary system without poles at the origin satisfying $M(\infty)=0$ and $N(s)$ be a strictly negative imaginary system. Then $[M(s), N(s)]$ is internally stable $\Leftrightarrow \bar{\lambda}[M(0) N(0)]<1$.

Proof: This is a direct consequence of Theorem 9 because:

1) $M(\infty)=0 \Rightarrow I-M(\infty) N(\infty)$ is nonsingular;

2) $\bar{\lambda}\left[[I-M(\infty) N(\infty)]^{-1}(M(\infty) N(0)-I)\right]=-1$ which means $\bar{\lambda}\left[[I-M(\infty) N(\infty)]^{-1}(M(\infty) N(0)-I)\right]<0$ is trivially fulfilled;

3) $\bar{\lambda}\left[[I-N(0) M(\infty)]^{-1}(N(0) M(0)-I)\right]<0$ if and only if $\bar{\lambda}[M(0) N(0)]<1$.

If in the proof of Theorem 9, we take the Schur complement around the other block of $T>0$, different necessary and sufficient conditions for the internal stability of a positive feedback interconnection of negative imaginary systems without poles at the origin can be derived as shown in Theorem 14. The matrices $(M(0) N(\infty)-I)[I-M(\infty) N(\infty)]^{-1}$ and $(N(0) M(0)-I)[I-N(\infty) M(0)]^{-1}$ in the conditions of Theorem 14 have only real eigenvalues due to Lemmas 3, 4 and 8.

Theorem 14: Let $M(s)$ be a negative imaginary system without poles at the origin and $N(s)$ be a strictly negative imaginary system. Then $[M(s), N(s)]$ is internally stable if and only if

$I-M(\infty) N(\infty)$ is nonsingular,

$$
\begin{aligned}
& \bar{\lambda}\left[(M(0) N(\infty)-I)[I-M(\infty) N(\infty)]^{-1}\right]<0, \text { and } \\
& \bar{\lambda}\left[(N(0) M(0)-I)[I-N(\infty) M(0)]^{-1}\right]<0 .
\end{aligned}
$$

Proof: The proof is identical to that in Theorem 9 until $T>0$. Then it proceeds in a similar way to the proof in Theorem 9 after $T>0$ by taking Schur complements about the $(1,1)$ element of $T>0$ instead of the $(2,2)$ element 
of $T>0$. Note that the conditions in Theorem 14 cannot be obtained directly via trivial algebraic manipulations of the conditions in Theorem 9 as shown in Lemma 3.

Example 2 demonstrates that if the closed-loop system is well-posed (i.e. $I-M(\infty) N(\infty)$ is nonsingular), each of the remaining conditions in Theorem 14 is not simply implied by either one of the remaining conditions in Theorem 9 independently.

Example 2: Choose $M(s)=\left[\begin{array}{cc}\frac{-s}{s+5} & \frac{-5}{s+5} \\ \frac{-(4 s+5)}{s^{2}+6 s+5} & \frac{-s^{2}+s+15}{s^{2}+6 s+5}\end{array}\right]$ and $N(s)=\left[\begin{array}{cc}\frac{5}{s+5} & \frac{-(2 s+15)}{s+5} \\ \frac{-\left(2 s^{2}+16 s+15\right)}{s^{2}+6 s+5} & \frac{-s^{2}+s+15}{s^{2}+6 s+5}\end{array}\right]$. Both $M(s)$ and $N(s)$ are strictly negative imaginary systems since $M(s)$ is strictly negative imaginary system from Example 1 and $N(s)=$ $M(s)+\left[\begin{array}{cc}1 & -2 \\ -2 & 0\end{array}\right]$. Also, the closed-loop system is wellposed, since $I-M(\infty) N(\infty)=\left[\begin{array}{cc}1 & -2 \\ -2 & 0\end{array}\right]$ is nonsingular. In this case, $\bar{\lambda}\left[[I-M(\infty) N(\infty)]^{-1}(M(\infty) N(0)-I)\right]=$ $-0.1340<0$ and $\bar{\lambda}\left[[I-N(0) M(\infty)]^{-1}(N(0) M(0)-I)\right]=$ $8 \nless 0$, whereas $\bar{\lambda}\left[(M(0) N(\infty)-I)[I-M(\infty) N(\infty)]^{-1}\right]=$ $3.3028 \nless 0$ and $\bar{\lambda}\left[(N(0) M(0)-I)[I-N(\infty) M(0)]^{-1}\right]=$ $1.5447 \nless 0$.

A different set of assumptions at 0 and $\infty$ frequencies can also be imposed to specialise the conditions of Theorem 14 , thereby obtaining another intuitive result that is different from Corollaries 12 and 13. Again note that the statement in Corollary 15 is also new and was not known in previous literature. In other words, it was not known that the dc loop gain condition is a necessary and sufficient condition for internal stability also under the simple assumption of $M(0)>0$ but no restriction of the sign of $N(\infty)$.

Corollary 15: Let $M(s)$ be a negative imaginary system without poles at the origin and $N(s)$ be a strictly negative imaginary system. Suppose $M(\infty) N(\infty)=0$ and $M(0)>0$. Then

$[M(s), N(s)]$ is internally stable $\Leftrightarrow \bar{\lambda}[M(0) N(0)]<1$.

Proof: This is a direct consequence of Theorem 14 because:

1) $M(\infty) N(\infty)=0$ implies $I-M(\infty) N(\infty)$ is nonsingular;

2) $\bar{\lambda}\left[(M(0) N(\infty)-I)[I-M(\infty) N(\infty)]^{-1}\right]<0$

$\Leftrightarrow \bar{\lambda}[M(0) N(\infty)]<1$

$\Leftrightarrow M(0)^{1 / 2} N(\infty) M(0)^{1 / 2}<I$

$\Leftrightarrow N(\infty)<M(0)^{-1}$

3) $\bar{\lambda}\left[(N(0) M(0)-I)[I-N(\infty) M(0)]^{-1}\right]<0$

$\Leftrightarrow \bar{\lambda}\left[\left(N(0)-M(0)^{-1}\right)\left[M(0)^{-1}-N(\infty)\right]^{-1}\right]<0$

$\Leftrightarrow \bar{\lambda}\left[\left[M(0)^{-1}-N(\infty)\right]^{-1 / 2}\left(N(0)-M(0)^{-1}\right)\left[M(0)^{-1}\right.\right.$

$\left.-N(\infty)]^{-1 / 2}\right]<0$

(since $M(0)^{-1}>N(\infty)$ via above)

$\Leftrightarrow N(0)-M(0)^{-1}<0$

$\Leftrightarrow \bar{\lambda}[M(0) N(0)]<1$.

But $\bar{\lambda}[M(0) N(0)]<1 \Leftrightarrow N(0)<M(0)^{-1} \Rightarrow N(\infty)<$ $M(0)^{-1}$ (since $N(\infty)<N(0)$ via [1, Lemma 2]).

This concludes the proof.

\section{SISO SPECIALISATION}

In Theorems 9 and 14, two different internal stability conditions are given. For the SISO case, these two stability conditions can be further reduced as shown in Lemma 16.

Lemma 16: Let $M(s)$ be a scalar negative imaginary system without poles at the origin and $N(s)$ be a scalar strictly negative imaginary system. Then the following three statements are equivalent:

1) $[M(s), N(s)]$ is internally stable;

2) either condition a) or condition b) holds:
a) $M(0) N(0)<1, M(\infty) N(\infty)<1$ and $M(\infty) N(0)<1$
b) $M(0) N(0)>1, M(\infty) N(\infty)>1$ and $M(\infty) N(0)>1$;

3) either condition a) or condition b) holds:
a) $M(0) N(0)<1, M(\infty) N(\infty)<1$ and $M(0) N(\infty)<1$
b) $M(0) N(0)>1, M(\infty) N(\infty)>1$ and $M(0) N(\infty)>1$.

Proof: Via Theorem 9, $[M(s), N(s)]$ is internally stable if and only if $M(\infty) N(\infty) \neq 1, \frac{1-M(\infty) N(0)}{M(\infty) N(\infty)-1}<0$, and $\frac{M(0) N(0)-1}{1-M(\infty) N(0)}<0$. These three conditions yield condition $\left.2 \mathrm{a}\right)$ or condition $2 b$ ).

Theorem 14 gives condition $3 a$ ) or condition $3 b$ ).

The internal stability conditions in Lemma 16 can be further simplified as shown in Theorem 17.

Theorem 17: Let $M(s)$ be a scalar negative imaginary system without poles at the origin and $N(s)$ be a scalar strictly negative imaginary system. Then $[M(s), N(s)]$ is internally stable if and only if either one of the following three conditions holds:

i) $M(0) N(0)<1$ and $M(\infty) N(\infty)<1$;

ii) $N(\infty)>0$ and $M(\infty) N(\infty)>1$;

iii) $N(0)<0$ and $M(0) N(0)>1$.

Proof: Both conditions 2a) and 3a) in Lemma 16 reduce to condition i) in this theorem statement via Lemma 5. This then implies that conditions $2 b$ ) and 3b) in Lemma 16 are equivalent.

Next recall that $N(0)>N(\infty)$ via [1, Lemma 2] and $M(0) \geq M(\infty)$ via Lemma 8 . Condition ii) and these two negative imaginary properties imply $N(0)>N(\infty)>0$ and $M(0) \geq M(\infty)>0$. Then $M(0) N(0)>M(0) N(\infty)$, $M(0) N(0) \geq M(\infty) N(0), M(\infty) N(0)>M(\infty) N(\infty)>1$ and $M(0) N(\infty) \geq M(\infty) N(\infty)>1$. These inequalities then imply conditions $2 b$ ) and 3b) in Lemma 16. Similarly, condition iii) and the same two negative imaginary properties imply $0>N(0)>N(\infty)$ and $0>$ $M(0) \geq M(\infty)$. Then $1<M(0) N(0)<M(0) N(\infty)$, $1<M(0) N(0) \leq M(\infty) N(0), M(\infty) N(0)<M(\infty) N(\infty)$ and $M(0) N(\infty) \leq M(\infty) N(\infty)$. These inequalities then imply conditions $2 b$ ) and $3 b$ ) in Lemma 16 .

To show the converse, consider the following five complimentary cases: $0<N(\infty)<N(0), 0=N(\infty)<N(0)$, $N(\infty)<0<N(0), N(\infty)<N(0)=0$, and $N(\infty)<$ $N(0)<0$. The three middle cases violate conditions 2 b) and $3 b$ ) in Lemma 16 . Hence only two valid complimentary 
cases are permitted by conditions $2 b$ ) and $3 b$ ) in Lemma 16: $0<N(\infty)$ and $N(0)<0$. Hence, condition 2 b) (respectively, condition 3b) ) in Lemma 16 implies either condition ii) or condition iii) of this theorem statement.

For the internal stability of the closed-loop system to be guaranteed via condition i) in Theorem 17, it is necessary and sufficient that the Nyquist plot of $M(s) N(s)$ starts and ends on the left-hand side of 1 . Recall that the Nyquist point is $+1+j 0$ since we are considering a positive feedback interconnection. Similarly, for the internal stability to be guaranteed by either conditions ii) or iii) in Theorem 17, it is necessary and sufficient that the Nyquist plot of $M(s) N(s)$ begins and ends on the right-hand side of 1 , and also that $M(0), M(\infty), N(0)$, and $N(\infty)$ have the same sign (positive in condition ii) and negative in condition iii) ).

The following example demonstrates use of Lemma 16 and Theorem 17.

Example 3: Choose $M(s)=\frac{3-s}{s+1}$ and $N(s)=\frac{1-2 s}{s+1}$. Both $M(s)$ and $N(s)$ are strictly negative imaginary systems. Hence they fulfill the assumptions of Lemma 16 and Theorem 17. In this case, $M(0) N(0)=(3)(1)>1$ and $M(\infty) N(\infty)=(-1)(-2)>1$, but $M(\infty) N(0)=$ $(-1)(1) \ngtr 1, M(0) N(\infty)=(3)(-2) \ngtr 1, N(\infty)=-2 \ngtr 0$ and $N(0)=1 \nless 0$. Lemma 16 and Theorem 17 correctly infer that $[M(s), N(s)]$ is not internally stable since $[I-M(s) N(s)]^{-1}=\frac{(s+1)^{2}}{-2+9 s-s^{2}}=\frac{-(s+1)^{2}}{s^{2}-9 s+2} \notin \mathscr{R} \mathscr{H}_{\infty}$.

Example 3 illustrates that although the positive frequency branch of the Nyquist plot of $M(s) N(s)$ begins and ends on the right-hand side of the Nyquist point $+1+j 0,[M(s), N(s)]$ is unstable since $M(0), M(\infty), N(0)$, and $N(\infty)$ have different signs. Indeed, $M(s) N(s)$ has no open-loop poles in the closed right half plane but the Nyquist plot of $M(s) N(s)$ encircles the Nyquist point $+1+j 0$ twice. Hence, there will be two unstable closed-loop poles which results in the instability of the closed-loop system according to the Nyquist stability criterion.

\section{Main Results: Part 2- Allowing Poles at the ORIGIN}

In the previous sections, we have discussed necessary and sufficient conditions for internal stability of the positive feedback interconnection shown in Fig. 1 involving a strictly negative imaginary system and a negative imaginary system without poles at the origin. Here, we derive more general results by allowing the negative imaginary system to have poles at the origin. To do this, we need to build on the results of the previous sections.

In the following subsections, we first provide several technical lemmas that underpin the subsequent theorems and results. Then, the general feedback stability results are derived. We also show how these general results specialise to the results in the earlier sections through simple algebraic manipulation and reduce to the results of [12] under the same assumptions as imposed in [12]. Lastly, we demonstrate that a matrix $\Psi$ with specific properties needed in the results always exists and propose a systematic way to construct a matrix $\Psi$. We also show that one such $\Psi$ is sufficient to conclude closed- loop stability, thereby we avoid having to search over the set of matrices $\Psi$ with certain properties.

\section{A. Technical Lemmas}

We introduce the following three technical lemmas as a basis for deriving the internal stability conditions in the next subsection. We first show that an original feedback system and a transformed system via a linear shift transformation have the same stability properties. Note that the loop transformation technique also holds even for the nonlinear systems [14, 26].

Lemma 18: Let $M(s)$ be a negative imaginary system and $N(s)$ be a strictly negative imaginary system. Let $\Psi<0$ be such that $I-M(\infty) \Psi$ is nonsingular. Define $M_{1}(s)=$ $[I-M(s) \Psi]^{-1} M(s)$ and $N_{1}(s)=N(s)-\Psi$. Then

1) $I-M(\infty) N(\infty)$ is nonsingular if and only if $I-$ $M_{1}(\infty) N_{1}(\infty)$ is nonsingular;

2) $[M(s), N(s)]$ is internally stable if and only if $\left[M_{1}(s), N_{1}(s)\right]$ is internally stable.

Proof:

1) $I-M(\infty) N(\infty)$ is nonsingular

$$
\begin{aligned}
\Leftrightarrow & I-M_{1}(\infty) N_{1}(\infty)=I-[I-M(\infty) \Psi]^{-1} M(\infty) \\
& (N(\infty)-\Psi)=[I-M(\infty) \Psi]^{-1}[I-M(\infty) N(\infty)] \text { is } \\
& \text { nonsingular }
\end{aligned}
$$

2) $[M(s), N(s)]$ is internally stable

$\Leftrightarrow I-M(\infty) N(\infty)$ is nonsingular (due to wellposedness condition) and $M(s)[I-N(s) M(s)]^{-1} \in$ $\mathscr{R} \mathscr{H}_{\infty}\left(\right.$ since $N(s) \in \mathscr{R} \mathscr{H}_{\infty}$, see [25, Corollary 5.4]) $\Leftrightarrow I-M_{1}(\infty) N_{1}(\infty)$ is nonsingular via 1) and $M_{1}(s)\left[I-N_{1}(s) M_{1}(s)\right]^{-1}=M(s)[I-\Psi M(s)]^{-1}$ $\left[I-(N(s)-\Psi) M(s)[I-\Psi M(s)]^{-1}\right]^{-1}=M(s)[I-$ $N(s) M(s)]^{-1} \in \mathscr{R} \mathscr{H}_{\infty}$

$\Leftrightarrow\left[M_{1}(s), N_{1}(s)\right]$ is internally stable.

In the next lemma we show that under a full normal rank assumption, a condition for a negative imaginary system to have no transmission zeros in the open right-half plane and at the origin is to choose the system gain at the infinity frequency to be positive semi-definite.

Lemma 19: Let $M(s)$ be a negative imaginary system with $M(\infty) \geq 0$. Suppose $M(s)$ has full normal rank. Then $M(s)$ has no transmission zeros in $\mathfrak{R}(s)>0$ and at the origin.

Proof: In this proof we use the notion of positive real systems as given by [2, Definition 1] and [2, Lemma 1]. We define the transfer function $\hat{M}(s)=M(s)-M(\infty)$ and $F(s)=s \hat{M}(s)$. Then $M(s)$ is negative imaginary if and only if $\hat{M}(s)$ is negative imaginary and $M(\infty)=M(\infty)^{T}$ via 1) in Lemma 7, and furthermore $\hat{M}(s)$ is negative imaginary if and only if $F(s)$ is positive real via 2) in Lemma 7. Then,

$$
\begin{aligned}
& M(\infty) \geq 0 \\
& \Rightarrow F_{1}(s)=F(s)+s M(\infty) \text { is positive real (since both } \\
& F(s) \text { and } s M(\infty) \text { are positive real and hence their } \\
& \text { addition must be positive real [27]) } \\
& \Leftrightarrow F_{1}(s)=s M(s) \text { is positive real } \\
& \Rightarrow F_{1}(s)^{-1}=\frac{1}{s} M(s)^{-1} \text { is positive real (since } F_{1}(s) \text { is } \\
& \text { positive real and } \exists s \in \mathbb{C}: \operatorname{det}\left[F_{1}(s)\right] \neq 0, \text { see }[27,
\end{aligned}
$$


Theorem 5-8]. So, $F_{1}(s)^{-1}$ has no poles in $\mathfrak{R}(s)>0$ and possibly has a simple pole at the origin)

$\Rightarrow M(s)^{-1}=s F_{1}(s)^{-1}$ has no poles in $\mathfrak{R}(s)>0$ and at the origin, since either $F_{1}(s)^{-1}$ has no pole at the origin or if $F_{1}(s)^{-1}$ has a simple pole at the origin then $s F_{1}(s)^{-1}$ has a pole-zero cancellation at the origin

$\Leftrightarrow M(s)$ has no transmission zeros in $\mathfrak{R}(s)>0$ and at the origin since $\exists s \in \mathbb{C}: \operatorname{det}[M(s)] \neq 0$.

The following technical lemma provides a condition for two transfer matrices to be marginally stable and have no poles at the origin.

Lemma 20: Let $M(s)$ be a negative imaginary system. Let $\Psi<0$ be such that $\bar{\lambda}[M(\infty) \Psi]<1$. Then

1) $[I-\Psi M(s)]^{-1}$ has no poles in $\mathfrak{R}(s)>0$ and at the origin;

2) $M_{1}(s)=M(s)[I-\Psi M(s)]^{-1}$ is a negative imaginary system without poles at the origin.

Proof:

$\bar{\lambda}[M(\infty) \Psi]<1$

$\Leftrightarrow \bar{\lambda}\left[(-\Psi)^{1 / 2}(-M(\infty))(-\Psi)^{1 / 2}\right]<1$

$\Leftrightarrow M(\infty)-\Psi^{-1}>0$

$\Rightarrow \operatorname{det}\left[M(\infty)-\Psi^{-1}\right] \neq 0$ and $M(s)-\Psi^{-1}$ has no transmission zeros in $\mathfrak{R}(s)>0$ and at $s=0$ via Lemma 19 since $M(s)-\Psi^{-1}$ is negative imaginary with $M(\infty)-\Psi^{-1}>0$ and $M(s)-\Psi^{-1}$ has full normal rank

$\Leftrightarrow[I-\Psi M(s)]^{-1}$ has no poles in $\Re(s)>0$ and at $s=0$ and $\operatorname{det}[I-\Psi M(\infty)] \neq 0$

$\Leftrightarrow-I+[I-\Psi M(s)]^{-1}$ has no poles in $\mathfrak{R}(s)>0$ and at $s=0$ and $\operatorname{det}[I-\Psi M(\infty)] \neq 0$

$\Leftrightarrow \Psi M(s)[I-\Psi M(s)]^{-1}$ has no poles in $\mathfrak{R}(s)>0$ and at $s=0$ and $\operatorname{det}[I-\Psi M(\infty)] \neq 0$

$\Leftrightarrow M_{1}(s)=M(s)[I-\Psi M(s)]^{-1}$ has no poles in $\Re(s)>$ 0 and at $s=0$ and $\operatorname{det}[I-\Psi M(\infty)] \neq 0$

$\Rightarrow M_{1}(s)=M(s)[I-\Psi M(s)]^{-1}$ is a negative imaginary system without poles at the origin via [2, Theorem 6] and since $M_{1}(s)$ has no poles at the origin. Note that the internal stability assumption in [2, Theorem 6] can be relaxed to analyticity in $\mathfrak{R}(s)>0$ in the non-strict negative imaginary setting.

The next lemma provides an equivalent condition for the maximum eigenvalue of the product of two symmetric matrices being less than unity.

Lemma 21: Let $A=A^{*} \in \mathbb{R}^{m \times m}, B=B^{*} \in \mathbb{R}^{m \times m}$, and $C=C^{*} \in \mathbb{R}^{m \times m}$ with $C<0$ and $A-B \geq 0$. Then $\bar{\lambda}[B C]<1$ if and only if $A-C^{-1}>0$ and $I-(A-B)^{1 / 2}(A-$ $\left.C^{-1}\right)^{-1}(A-B)^{1 / 2}>0$.

Proof:

$\bar{\lambda}[B C]<1$

$\Leftrightarrow \bar{\lambda}\left[(-C)^{1 / 2}(-B)(-C)^{1 / 2}\right]<1($ via $C<0)$

$\Leftrightarrow-C^{-1}+B>0$

$\Leftrightarrow\left(A-C^{-1}\right)-(A-B)>0$

$\Leftrightarrow\left[\begin{array}{cc}A-C^{-1} & (A-B)^{1 / 2} \\ (A-B)^{1 / 2} & I\end{array}\right]>0($ via $A-B \geq 0)$

$\Leftrightarrow A-C^{-1}>0$ and

$$
I-(A-B)^{1 / 2}\left(A-C^{-1}\right)^{-1}(A-B)^{1 / 2}>0 .
$$

The next two highly technical lemmas below are used to underpin the results in Subsection V-D. We provide a rewritten expression for $\lambda_{i}[(-I+A C)(I-B C)]$ in the following lemma.

Lemma 22: Let $A, B$ and $C$ be real symmetric matrices with $C \geq 0$. Then $\lambda_{i}[(-I+A C)(I-B C)]=\lambda_{i}[(-I+$ $\left.\left.C^{1 / 2} A C^{1 / 2}\right)\left(I-C^{1 / 2} B C^{1 / 2}\right)\right]$ for all $i$.

Proof:

$$
\begin{aligned}
\lambda_{i}[(-I & +A C)(I-B C)] \\
& =\lambda_{i}\left[C^{1 / 2}(A+B-A C B) C^{1 / 2}\right]-1 \quad(\text { via } C \geq 0) \\
& =\lambda_{i}\left[\left(-I+C^{1 / 2} A C^{1 / 2}\right)\left(I-C^{1 / 2} B C^{1 / 2}\right)\right] .
\end{aligned}
$$

The positiveness and positive semidefiniteness of the transfer matrices are shown in the following lemma via algebraic manipulation with specific choices of the $\Psi$.

Lemma 23: Let $M(s)$ be a negative imaginary system. Let $\Psi<0$ be such that $\bar{\lambda}[M(\infty) \Psi]<1$. Define $Q=[M(\infty)-$ $\left.\Psi^{-1}\right]^{1 / 2}\left(I-\left[M(\infty)-\Psi^{-1}\right]^{1 / 2} \lim _{s \rightarrow 0}\left[M(s)-\Psi^{-1}\right]^{-1}[M(\infty)-\right.$ $\left.\left.\Psi^{-1}\right]^{1 / 2}\right)\left[M(\infty)-\Psi^{-1}\right]^{1 / 2}$. Then

1) $M(\infty)-\Psi^{-1}>0$;

2) $\lim _{s \rightarrow 0}\left[M(s)-\Psi^{-1}\right]^{-1} \geq 0$;

3) $\stackrel{s \rightarrow 0}{Q \geq 0}$;

4) $I-Q^{1 / 2}\left[M(\infty)-\Psi^{-1}\right]^{-1} Q^{1 / 2} \geq 0$.

Furthermore, let $\Psi_{1}<0$ be such that $\bar{\lambda}\left[M(\infty) \Psi_{1}\right]<1$ and define $M_{1}(s)=M(s)[I-\Psi M(s)]^{-1}$. Then,

5) $\lim _{s \rightarrow 0}\left[\left[M(s)-\Psi^{-1}\right]\left[M(s)-\Psi_{1}^{-1}\right]^{-1}\right]$ is nonsingular;

6) $I-\left[M_{1}(0)-M_{1}(\infty)\right]^{1 / 2}\left[I+\left(\Psi-\Psi_{1}\right) M_{1}(0)\right]^{-1}(\Psi-$ $\left.\Psi_{1}\right)\left[M_{1}(0)-M_{1}(\infty)\right]^{1 / 2}>0$.

Proof:

1) $\bar{\lambda}[M(\infty) \Psi]<1$

$$
\Leftrightarrow \bar{\lambda}\left[(-\Psi)^{1 / 2}[-M(\infty)](-\Psi)^{1 / 2}\right]<1
$$$$
\Leftrightarrow M(\infty)-\Psi^{-1}>0
$$

2) $\lim _{s \rightarrow 0}\left[M(s)-\Psi^{-1}\right]^{-1}$

$=\lim _{s \rightarrow 0}\left[[M(s)-M(\infty)]+\left[M(\infty)-\Psi^{-1}\right]\right]^{-1}$

$=\lim _{s \rightarrow 0} s\left[s[M(s)-M(\infty)]+s\left[M(\infty)-\Psi^{-1}\right]\right]^{-1} \geq 0$ (since both $s[M(s)-M(\infty)]$ and $s\left[M(\infty)-\Psi^{-1}\right]$ are positive real via Lemma 7 and 1), hence their addition must be positive real [27] and since $s[M(s)-$ $M(\infty)]+s\left[M(\infty)-\Psi^{-1}\right]$ is positive real, its inverse is also positive real [27, Theorem 5-8]. If $[s[M(s)-$ $\left.M(\infty)]+s\left[M(\infty)-\Psi^{-1}\right]\right]^{-1}$ has a simple pole at the origin, then its residue is Hermitian and positive semidefinite. Otherwise, $\lim _{s \rightarrow 0} s[s[M(s)-M(\infty)]+$ $\left.\left.s\left[M(\infty)-\Psi^{-1}\right]\right]^{-1}=0\right)$

3) $M(s)$ is a negative imaginary system

$\Rightarrow M(s)[I-\Psi M(s)]^{-1}$ is a negative imaginary system without poles at the origin, via 2) in Lemma 20

$\Rightarrow \lim _{s \rightarrow 0} M(s)[I-\Psi M(s)]^{-1}-M(\infty)[I-\Psi M(\infty)]^{-1}$ $\stackrel{s \rightarrow 0}{\geq 0}($ via Lemma 8$)$

$\Leftrightarrow \underline{\lambda}\left[-\lim _{s \rightarrow 0}[I-\Psi M(s)]^{-1}+[I-\Psi M(\infty)]^{-1}\right] \geq 0$ 
$\Leftrightarrow I-\left[M(\infty)-\Psi^{-1}\right]^{1 / 2} \lim _{s \rightarrow 0}\left[M(s)-\Psi^{-1}\right]^{-1}[M(\infty)-$ $\left.\Psi^{-1}\right]^{1 / 2} \geq 0\left(\right.$ since $M(\infty)-\Psi^{-1}>0$ via 1$)$ ) $\Leftrightarrow Q \geq 0$

4) $\lim _{s \rightarrow 0}\left[M(s)-\Psi^{-1}\right]^{-1} \geq 0$ (via 2))

$\Leftrightarrow \underline{\lambda}\left[\lim _{s \rightarrow 0}\left[M(s)-\Psi^{-1}\right]^{-1}\left[M(\infty)-\Psi^{-1}\right]\right] \geq 0$

$\Leftrightarrow \underline{\lambda}\left[I-\left[M(\infty)-\Psi^{-1}\right]^{-1} Q\right] \geq 0$

$\Leftrightarrow I-Q^{1 / 2}\left[M(\infty)-\Psi^{-1}\right]^{-1} Q^{1 / 2} \geq 0$ (since $Q \geq 0$ via 3$)$ ).

5) For convenience, define $\Psi_{2}<0$ such that $\Psi_{2}{ }^{-1}<\Psi^{-1}$ and $\Psi_{2}{ }^{-1}<\Psi_{1}{ }^{-1}$. Then

$$
\begin{aligned}
& \lim _{s \rightarrow 0}\left[\left[M(s)-\Psi^{-1}\right]\left[M(s)-\Psi_{1}{ }^{-1}\right]^{-1}\right] \\
&=\lim _{s \rightarrow 0}\left[\left[M(s)-\Psi^{-1}\right]\left[M(s)-\Psi_{2}^{-1}\right]^{-1}\left[M(s)-\Psi_{2}^{-1}\right]\right. \\
&\left.=\left[M(s)-\Psi_{1}^{-1}\right]^{-1}\right] \\
&=\left[\left(\Psi^{-1}-\Psi_{2}{ }^{-1}\right)^{-1}+\lim _{s \rightarrow 0}\left[M(s)-\Psi^{-1}\right]^{-1}\right]^{-1}\left(\Psi^{-1}-\right. \\
&\left.\Psi_{2}{ }^{-1}\right)^{-1}\left(\Psi_{1}{ }^{-1}-\Psi_{2}^{-1}\right)\left[\left(\Psi_{1}^{-1}-\Psi_{2}{ }^{-1}\right)^{-1}+\right. \\
&\left.\lim _{s \rightarrow 0}\left[M(s)-\Psi_{1}^{-1}\right]^{-1}\right] \text { is nonsingular }\left(\text { since } \Psi^{-1}-\right. \\
& \Psi_{2}{ }^{-1}>0, \Psi_{1}^{-1}-\Psi_{2}^{-1}>0, \lim _{s \rightarrow 0}\left[M(s)-\Psi^{-1}\right]^{-1} \geq \\
&\left.\left.0, \text { and } \lim _{s \rightarrow 0}\left[M(s)-\Psi_{1}{ }^{-1}\right]^{-1} \geq 0 \text { via } 2\right)\right)
\end{aligned}
$$

6) For convenience, define $Q_{1}=\left[M(\infty)-\Psi_{1}^{-1}\right]^{1 / 2}(I-$ $\left[M(\infty)-\Psi_{1}^{-1}\right]^{1 / 2} \lim _{s \rightarrow 0}\left[M(s)-\Psi_{1}^{-1}\right]^{-1}[M(\infty)-$ $\left.\left.\Psi_{1}^{-1}\right]^{1 / 2}\right)\left[M(\infty)-{\stackrel{s \rightarrow 0}{\Psi_{1}^{-1}}}^{1 / 2}\right.$. Then

$I-Q_{1}^{1 / 2}\left[M(\infty)-\Psi_{1}^{-1}\right]^{-1} Q_{1}^{1 / 2}+Q_{1}^{1 / 2}[M(\infty)-$ $\left.\Psi^{-1}\right]^{-1} Q_{1}^{1 / 2} \geq 0$ (since $\left[M(\infty)-\Psi^{-1}\right]^{-1}>0$ and $Q_{1} \geq$ 0 via 1) and 3) and $I-Q_{1}^{1 / 2}\left[M(\infty)-\Psi_{1}^{-1}\right]^{-1} Q_{1}^{1 / 2} \geq 0$ via 4))

$$
\begin{aligned}
\Leftrightarrow & \underline{\lambda}\left[\operatorname { l i m } _ { s \rightarrow 0 } \left[I+\left[\left[M(\infty)-\Psi^{-1}\right]^{-1}-\left[M(\infty)-\Psi_{1}^{-1}\right]^{-1}\right]\right.\right. \\
& {[M(s)-M(\infty)]\left[I+\left[M(\infty)-\Psi^{-1}\right]^{-1}[M(s)-\right.} \\
& \left.\left.M(\infty)]]^{-1}\right]\right] \geq 0 \\
\Leftrightarrow & \underline{\lambda}\left[\operatorname { l i m } _ { s \rightarrow 0 } \left[\left[I+\left[M(\infty)-\Psi^{-1}\right]^{-1}[M(s)-M(\infty)]\right][I+\right.\right. \\
& {\left.\left.\left.\left[M(\infty)-\Psi_{1}{ }^{-1}\right]^{-1}[M(s)-M(\infty)]\right]^{-1}\right]\right] \geq 0 } \\
\Leftrightarrow & \underline{\lambda}\left[\lim _{s \rightarrow 0}\left[M(\infty)-\Psi^{-1}\right]^{-1}\left[M(s)-\Psi^{-1}\right][M(s)-\right. \\
& \left.\left.\left.\left.\left.\Psi_{1}^{-1}\right]^{-1}\left[M(\infty)-\Psi_{1}^{-1}\right]\right]\right]>0 \text { via } 1\right) \text { and } 5\right) \\
\Leftrightarrow & I-\left[M_{1}(0)-M_{1}(\infty)\right]^{1 / 2}\left[I+\left(\Psi-\Psi_{1}\right) M_{1}(0)\right]^{-1}(\Psi \\
& \left.-\Psi_{1}\right)\left[M_{1}(0)-M_{1}(\infty)\right]^{1 / 2}>0 .
\end{aligned}
$$

\section{B. Generalised Internal Stability Results for Negative Imagi- nary Systems with Possible Poles at the Origin}

In this subsection, necessary and sufficient conditions are derived for internal stability of a positive feedback interconnection composed of a negative imaginary system (which may or may not have poles at the origin) and a strictly negative imaginary system. The limits in the stability conditions will be shown to be all finite. We obtain the general result in Theorem 24 by building on Theorem 9, which was only applicable for negative imaginary systems without poles at the origin, by using an appropriate linear shift transformation to remove the undesirable restriction of no poles at the origin. This then enables Theorem 24 to be also applicable for negative imaginary systems which may or may not have free body dynamics (i.e. poles at the origin).
Theorem 24: Let $M(s)$ be a negative imaginary system and $N(s)$ be a strictly negative imaginary system. Let $\Psi<0$ be such that $\bar{\lambda}[M(\infty) \Psi]<1$. Then $[M(s), N(s)]$ is internally stable if and only if

$$
I-M(\infty) N(\infty) \text { is nonsingular, }
$$

$\bar{\lambda}\left[[I-M(\infty) N(\infty)]^{-1}[M(\infty) N(0)-I]\right]<0$, and

$\bar{\lambda}\left[\lim _{s \rightarrow 0}\left[[I-\Psi M(\infty)][I-N(s) M(\infty)]^{-1}[N(s) M(s)-I]\right.\right.$

$\left.\left.[I-\Psi M(s)]^{-1}\right]\right]<0$.

Proof: Define $M_{1}(s)=[I-M(s) \Psi]^{-1} M(s)$ and $N_{1}(s)=N(s)-\Psi$. Then,

$[M(s), N(s)]$ is internally stable

$\Leftrightarrow\left[M_{1}(s), N_{1}(s)\right]$ is internally stable (by Lemma 18)

$\Leftrightarrow I-M_{1}(\infty) N_{1}(\infty)$ is nonsingular, $\bar{\lambda}\left[\left[I-M_{1}(\infty) N_{1}(\infty)\right]^{-1}\left(M_{1}(\infty) N_{1}(0)-I\right)\right]<0$, and $\bar{\lambda}\left[\left[I-N_{1}(0) M_{1}(\infty)\right]^{-1}\left(N_{1}(0) M_{1}(0)-I\right)\right]<0$ (since $M_{1}(s)$ is a negative imaginary system without poles at the origin via Lemma 20 and $N_{1}(s)$ is a strictly negative imaginary system and then direct use of Theorem 9)

$\Leftrightarrow I-M(\infty) N(\infty)$ is nonsingular (via Lemma 18),

$\bar{\lambda}\left[\left[I-[I-M(\infty) \Psi]^{-1} M(\infty)(N(\infty)-\Psi)\right]^{-1}[[I-\right.$ $\left.\left.M(\infty) \Psi]^{-1} M(\infty)(N(0)-\Psi)-I\right]\right]<0$, and $\bar{\lambda}\left[\lim _{s \rightarrow 0}\left[\left[I-(N(s)-\Psi)[I-M(\infty) \Psi]^{-1} M(\infty)\right]^{-1}\right.\right.$ $\left.\left.\left[(\stackrel{s \rightarrow 0}{N}(s)-\Psi)[I-M(s) \Psi]^{-1} M(s)-I\right]\right]\right]<0$

$\Leftrightarrow I-M(\infty) N(\infty)$ is nonsingular,

$\bar{\lambda}\left[\left[[I-M(\infty) \Psi]^{-1}[I-M(\infty) \Psi-M(\infty) N(\infty)+\right.\right.$ $M(\infty) \Psi]]^{-1}\left[[I-M(\infty) \Psi]^{-1}[M(\infty) N(0)-M(\infty)\right.$ $\Psi-I+M(\infty) \Psi]]]<0$, and

$\bar{\lambda}\left[\lim _{s \rightarrow 0}\left[\left[I-(N(s)-\Psi) M(\infty)[I-\Psi M(\infty)]^{-1}\right]^{-1}\right.\right.$ $\left.\left.\left[(N(s)-\Psi) M(s)[I-\Psi M(s)]^{-1}-I\right]\right]\right]<0$

$\Leftrightarrow I-M(\infty) N(\infty)$ is nonsingular,

$\bar{\lambda}\left[[I-M(\infty) N(\infty)]^{-1}[I-M(\infty) \Psi][I-M(\infty) \Psi]^{-1}\right.$ $[M(\infty) N(0)-I]]<0$, and $\bar{\lambda}\left[\lim _{s \rightarrow 0}[[[I-\Psi M(\infty)-N(s) M(\infty)+\Psi M(\infty)][I-\right.$ $\left.\Psi M(\infty)]^{-1}\right]^{-1}[[N(s) M(s)-\Psi M(s)-I+\Psi M(s)]$ $\left.\left.\left.[I-\Psi M(s)]^{-1}\right]\right]\right]<0$

$\Leftrightarrow I-M(\infty) N(\infty)$ is nonsingular,

$\bar{\lambda}\left[[I-M(\infty) N(\infty)]^{-1}[M(\infty) N(0)-I]\right]<0$, and $\bar{\lambda}\left[\lim _{s \rightarrow 0}\left[[I-\Psi M(\infty)][I-N(s) M(\infty)]^{-1}[N(s) M(s)\right.\right.$ $\left.\left.-I][I-\Psi M(s)]^{-1}\right]\right]<0$.

The above algebraic reformulations, together with Lemmas 4 and 8 , also demonstrate why the eigenvalues of the matrices in the conditions of the theorem statement are real.

Remark 25: We need to show that $\lim _{s \rightarrow 0}[[N(s) M(s)-$ $\left.I][I-\Psi M(s)]^{-1}\right]=\lim _{s \rightarrow 0} N(s) M(s)[I \stackrel{s \rightarrow 0}{-} \Psi M(s)]^{-1}-$ $\lim _{s \rightarrow 0}[I-\Psi M(s)]^{-1}$ is finite. Since both $M(s)[I-\Psi M(s)]^{-1}$ and $[I-\Psi M(s)]^{-1}$ have no poles at the origin via Lemma 20, $\lim _{s \rightarrow 0}\left[[N(s) M(s)-I][I-\Psi M(s)]^{-1}\right]$ is finite.

We now give a different internal stability necessary and sufficient condition in Theorem 26 which is based on Theorem 14 instead of Theorem 9.

Theorem 26: Let $M(s)$ be a negative imaginary system and $N(s)$ be a strictly negative imaginary system. Let $\Psi<0$ be such that $\bar{\lambda}[M(\infty) \Psi]<1$. Then $[M(s), N(s)]$ is internally stable if and only if 
$I-M(\infty) N(\infty)$ is nonsingular,

$\bar{\lambda}\left[\lim _{s \rightarrow 0}\left[[I-M(s) \Psi]^{-1}[M(s) N(\infty)-I][I-M(\infty)\right.\right.$

$\left.\left.N(\infty)]^{-1}[I-M(\infty) \Psi]\right]\right]<0$, and

$\bar{\lambda}\left[\lim _{s \rightarrow 0}\left[[N(s) M(s)-I][I-N(\infty) M(s)]^{-1}\right]\right]<0$.

Proof: The proof is similar to that of Theorem 24 except Theorem 14 is used instead of Theorem 9.

Remark 27: We need to show that $\lim _{s \rightarrow 0}\left[[I-M(s) \Psi]^{-1}\right.$ $\left.[M(s) N(\infty)-I][I-M(\infty) N(\infty)]^{-1}[I-M(\infty) \Psi]\right]$ is finite. Note that $\lim _{s \rightarrow 0}\left[[I-M(s) \Psi]^{-1}[M(s) N(\infty)-I]\right]$ is finite by Lemma $20,(I-M(\infty) N(\infty))^{-1}$ is finite via the first condition in Theorem 26 and $I-M(\infty) \Psi$ is obviously finite.

The following technical lemma will be needed in Remark 29 to show that the limit in the third condition of Theorem 26 is also finite.

Lemma 28: Let all the assumptions in Theorem 26 hold. Furthermore, suppose that $I-M(\infty) N(\infty)$ is nonsingular and $\bar{\lambda}\left[\lim _{s \rightarrow 0}\left[[I-M(s) \Psi]^{-1}[M(s) N(\infty)-I][I-\right.\right.$ $\left.\left.M(\infty) N(\infty)]^{-1}[I-M(\infty) \Psi]\right]\right]<0$. Then $\lim _{s \rightarrow 0}[[I-$ $\left.\Psi M(s)][I-N(\infty) M(s)]^{-1}\right]$ is finite and nonsingular.

Proof: First note that $\lim _{s \rightarrow 0}[[N(\infty) M(s)-I][I-$ $\left.\Psi M(s)]^{-1}\right]$ is finite via Lemma 20 . Next, note that

$\bar{\lambda}\left[\lim _{s \rightarrow 0}\left[[I-M(s) \Psi]^{-1}[M(s) N(\infty)-I][I-M(\infty) N(\infty)]^{-1}\right.\right.$ $[I-M(\infty) \Psi]]]<0$

$\Rightarrow \lim _{s \rightarrow 0}\left[[I-M(s) \Psi]^{-1}[M(s) N(\infty)-I][I-M(\infty)\right.$ $\left.N(\infty)]^{-1}[I-M(\infty) \Psi]\right]$ is nonsingular

$\Rightarrow \lim _{s \rightarrow 0}\left[[I-M(s) \Psi]^{-1}[M(s) N(\infty)-I]\right]$ is nonsingular

$\Rightarrow \lim _{s \rightarrow 0} \frac{\operatorname{det}(M(s) N(\infty)-I)}{\operatorname{det}(I-M(s) \Psi)} \neq 0$

$\Rightarrow \lim _{s \rightarrow 0} \frac{\operatorname{det}(N(\infty) M(s)-I)}{\operatorname{det}(I-\Psi M(s))} \neq 0$

$\Rightarrow \lim _{s \rightarrow 0}\left[[N(\infty) M(s)-I][I-\Psi M(s)]^{-1}\right]$ is nonsingular.

Then

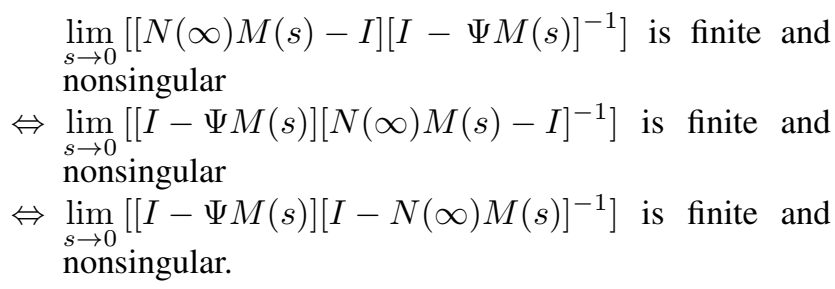

Remark 29: We need to show that $\lim _{s \rightarrow 0}[[N(s) M(s)-I][I-$ $\left.N(\infty) M(s)]^{-1}\right]=\lim _{s \rightarrow 0}\left[[N(s) M(s)-I][I-\Psi M(s)]^{-1}[I-\right.$ $\left.\Psi M(s)][I-N(\infty) M(s)]^{-1}\right]$ is finite. It is clear that $\lim _{s \rightarrow 0}\left[[N(s) M(s)-I][I-\Psi M(s)]^{-1}\right]$ and $\lim _{s \rightarrow 0}[[I-\Psi M(s)]$ $\left.[I-N(\infty) M(s)]^{-1}\right]$ are finite via Lemmas 20 and 28 respectively. Hence, $\lim _{s \rightarrow 0}\left[[N(s) M(s)-I][I-N(\infty) M(s)]^{-1}\right]$ is finite.

In contrast with the stability results in the literature $[1,11$, 12, 23], the conditions presented here are a generalisation on those results in at least three aspects: 1) the stability results allow for poles on the imaginary axis and at the origin, 2) the stability results impose no restriction on the instantaneous gains of the systems, and 3) the conditions are significantly easier to check. Note that [12] imposes several restrictive assumptions, including strict properness of the negative imaginary system, and requires multiple complex matrix factorisations. Furthermore, some of the assumptions in [12] are imposed on the resulting factorisations, thereby destroying intuition. The results in Theorems 24 and 26 impose no such restrictions. Subsection V-D discusses existence and construction of the matrix $\Psi$ that is needed in Theorems 24 and 26 .

\section{Specialisations of the Generalised Internal Stability Con- ditions}

First, we give a corollary that does not depend on the matrix $\Psi$. It is a SISO specialisation of both Theorems 24 and 26 in the special situation when the negative imaginary system necessarily has poles at the origin.

Corollary 30: Let $M(s)$ be a scalar, negative imaginary system and $N(s)$ be a scalar, strictly negative imaginary system. Let $s=0$ be a (single or double) pole of $M(s)$. Then $[M(s), N(s)]$ is internally stable if and only if either one of the following two conditions holds:

i) $M(\infty) N(\infty)<1$ and $N(0)<0$;

ii) $M(\infty) N(\infty)>1$ and $N(\infty)>0$.

Proof: Writing $M(s)$ as a Laurent series, the three conditions in Theorem 26 simplify, in the scalar case, to $M(\infty) N(\infty) \neq 1, \frac{N(\infty)}{1-M(\infty) N(\infty)}<0$ and $\frac{N(0)}{N(\infty)}>0$ after taking the limit as $s \rightarrow 0$. It is easy to see that these three conditions are equivalent to either condition i) or condition ii) in this corollary statement using the negative imaginary property $N(0)>N(\infty)$ via [1, Lemma 2].

Alternatively, Theorem 24 can be used instead of Theorem 26 to give the same specialisations. Writing $M(s)$ as a Laurent series, the three conditions in Theorem 24 simplify, in the scalar case, to $M(\infty) N(\infty) \neq 1, \frac{M(\infty) N(0)-1}{1-M(\infty) N(\infty)}<0$ and $\frac{N(0)}{1-N(0) M(\infty)}<0$ after taking the limit as $s \rightarrow 0$. These three conditions are equivalent to either one of the following two statements:

1) $M(\infty) N(\infty)<1, M(\infty) N(0)<1$ and $N(0)<0$;

2) $M(\infty) N(\infty)>1, M(\infty) N(0)>1$ and $N(0)>0$.

Equivalence between condition 1) and condition i) in the corollary statement can be seen on noting that $M(\infty) N(0)=$ $[M(\infty) N(\infty)]\left[\frac{N(0)}{N(\infty)}\right]<1$ since $N(\infty)<N(0)<0$ via [1, Lemma 2]. Equivalence between condition 2) and condition ii) in the corollary statement can be seen on noting that $M(\infty) N(0)=[M(\infty) N(\infty)]\left[\frac{N(0)}{N(\infty)}\right]>1$ since $0<N(\infty)<N(0)$ via [1, Lemma 2].

Next, we specialise Theorems 24 and 26 to a MIMO case that is in fact still a generalisation of the results in [12]. If we consider the situation that $M(s)$ is a strictly proper transfer function with negative imaginary properties, then Theorems 24 and 26 can be specialised to the results in 2) and 3) in Corollary 31 respectively.

Corollary 31: Let $M(s)$ be a strictly proper, negative imaginary system and $N(s)$ be a strictly negative imaginary system. Let $\Psi<0$. Then, the following three conditions are equivalent: 
1) $[M(s), N(s)]$ is internally stable;

2) $\bar{\lambda}\left[\lim _{s \rightarrow 0}\left[[N(s) M(s)-I][I-\Psi M(s)]^{-1}\right]\right]<0$;

3) $\bar{\lambda}\left[\lim _{s \rightarrow 0}\left[[I-M(s) \Psi]^{-1}[M(s) N(\infty)-I]\right]\right]<0$ and $\bar{\lambda}\left[\lim _{s \rightarrow 0}\left[[N(s) M(s)-I][I-N(\infty) M(s)]^{-1}\right]\right]<0$.

Proof: The equivalence between 1) and 2) is omitted since it is the direct consequence of Theorem 24 and the assumption of strictly properness of $M(s)$. The equivalence between 1) and 3) is omitted since it is a direct consequence of Theorem 26 and the assumption of strict properness of $M(s)$.

Note that the limit in 2) of Corollary 31 is finite via Lemma 20 and the limits in 3) of Corollary 31 are finite via Lemmas 20 and 28. Under the same assumption of strictly proper $M(s)$, [12] proposed necessary and sufficient conditions for internal stability of positive feedback interconnections of negative imaginary systems. However, the stability results in [12] require additional assumptions such as the invertibility of $F^{T} \bar{G}(0) F$ and $N_{f}$ being either positive or negative definite (see [12, Thereom 1]). When these assumptions imposed in [12] do not hold, such as $F^{T} \bar{G}(0) F$ being singular or $N_{f}$ being sign-indefinite, the conditions in [12] are no longer applicable. Unlike the results in [12], the conditions in Corollary 31 are easy-to-check, are necessary and sufficient conditions, impose no extra assumptions, and do not require any matrix factorisations. Indeed, the extra assumptions in [12] on objects that are a result of such factorisations unfortunately inhibit intuition into the results of [12].

The internal stability condition in 2) of Corollary 31 only depends on the steady-state (or DC) gains and a negative definite matrix $\Psi$. If we rearrange the inequality in 2 ), we end up to have $\bar{\lambda}\left[M_{1}(0) N_{1}(0)\right]<1$, where $M_{1}(s)=[I-$ $M(s) \Psi]^{-1} M(s)$ and $N_{1}(s)=N(s)-\Psi$, which means that the stability of the positive-feedback interconnection relies on only the dc loop gain of the transformed system. Surprisingly, simplification of the two inequalities in 3) to obtain the inequality in 2) is not directly obvious for the MIMO situation. However, for the SISO case, it is straightforward to realise that the terms of $[M(s) N(\infty)-I]$ and $[I-N(\infty) M(s)]^{-1}$ in first and second inequalities of 3 ) can be eliminated via multiplying both inequalities in 3) to give the inequality in 2).

We next give a corollary that does not depend of the matrix $\Psi$. It is a MIMO specialisation of Corollary 31 in the situation when the negative imaginary system necessarily has single or double poles at the origin in all directions.

Corollary 32: Let $M(s)$ be a strictly proper, negative imaginary system and $N(s)$ be a strictly negative imaginary system. Assume one of the following conditions holds:

- $\lim _{s \rightarrow 0} s^{2} M(s)$ is nonsingular;

- $\lim _{s \rightarrow 0} s^{2} M(s)=0$ and $\lim _{s \rightarrow 0} s M(s)$ is nonsingular.

Then $[M(s), N(s)]$ is internally stable if and only if $N(0)<0$.

Proof: Consider each of the two cases of this corollary separately. Writing $M(s)$ as a Laurent series into either condition 2) or condition 3) of Corollary 31 gives the required result after evaluating the limit and simplifying.

In Subsection V-B, general internal stability conditions of positive feedback interconnections of negative imaginary systems were derived. We now show that the main stability theorems of that subsection (i.e. Theorems 24 and 26) specialise to the prior stability conditions in Theorems 9 and 14 respectively under the corresponding assumption that $M(s)$ is a negative imaginary system without poles at the origin, thereby demonstrating the generality of Theorems 24 and 26 .

Since, by inspection, two of the conditions in Theorem 24 (respectively Theorem 26) are trivially equivalent to two of the conditions in Theorem 9 (respectively Theorem 14), we only need to proof equivalence of the remaining inequality in Theorem 24 (respectively Theorem 26) with the remaining inequality in Theorem 9 (respectively Theorem 14). Note that to show these equivalences in Lemmas 33 and 34, we cannot simply assume that $\Psi=0$ since $\Psi<0$ is required in the assumptions of both Theorems 24 and 26 and a limiting argument cannot be used as the results in Theorems 24 and 26 are valid for any arbitrary $\Psi<0$ that satisfies $\bar{\lambda}(M(\infty) \Psi)<1$.

Lemma 33: Let all the assumptions of Theorem 24 hold and furthermore suppose $M(s)$ has no poles at the origin. Then

$$
\begin{aligned}
& \bar{\lambda}\left[\operatorname { l i m } _ { s \rightarrow 0 } \left[[I-\Psi M(\infty)][I-N(s) M(\infty)]^{-1}[N(s) M(s)\right.\right. \\
& \left.\left.-I][I-\Psi M(s)]^{-1}\right]\right]<0 \\
\Leftrightarrow & \bar{\lambda}\left[[I-N(0) M(\infty)]^{-1}(N(0) M(0)-I)\right]<0 .
\end{aligned}
$$

Proof: Let $R=[M(0)-M(\infty)]^{1 / 2}$. See Lemma 8 for proof that $M(0)-M(\infty) \geq 0$. Then,

$$
\begin{aligned}
& \bar{\lambda}\left[\operatorname { l i m } _ { s \rightarrow 0 } \left[[I-\Psi M(\infty)][I-N(s) M(\infty)]^{-1}[N(s) M(s)-I][I-\right.\right. \\
&\left.\left.\Psi M(s)]^{-1}\right]\right]<0 \\
& \Leftrightarrow \bar{\lambda}\left[[I-\Psi M(\infty)][I-N(0) M(\infty)]^{-1}[N(0) M(0)-I][I-\right. \\
&\left.\Psi M(0)]^{-1}\right]<0 \text { (since } M(s) \text { is a negative imaginary } \\
& \text { system without poles at the origin and } N(s) \text { is a strictly } \\
&\text { negative imaginary system, } M(0) \text { and } N(0) \text { exist }) \\
& \Leftrightarrow \bar{\lambda}\left[[I-\Psi M(0)]^{-1}[I-\Psi M(\infty)][I-N(0) M(\infty)]^{-1}\right. \\
& {[N(0) M(0)-I]<0 } \\
& \Leftrightarrow \bar{\lambda}\left[[I-\Psi M(0)]^{-1}[I-\Psi M(0)+\Psi M(0)-\Psi M(\infty)]\right. \\
& {[I-N(0) M(\infty)]^{-1}[N(0) M(0)-N(0) M(\infty)+N(0)} \\
&M(\infty)-I]]<0 \\
& \Leftrightarrow \bar{\lambda}\left[[ I + [ I - \Psi M ( 0 ) ] ^ { - 1 } \Psi R ^ { 2 } ] \left[[I-N(0) M(\infty)]^{-1} N(0)\right.\right. \\
&\left.\left.R^{2}-I\right]\right]<0 \\
& \Leftrightarrow \bar{\lambda}\left[\left[[I-N(0) M(\infty)]^{-1} N(0)-[I-\Psi M(0)]^{-1} \Psi+[I-\right.\right. \\
&\left.\left.\Psi M(0)]^{-1} \Psi R^{2}[I-N(0) M(\infty)]^{-1} N(0)\right] R^{2}\right]<1 \\
& \Leftrightarrow \bar{\lambda}\left[R \left[[I-N(0) M(\infty)]^{-1} N(0)-[I-\Psi M(0)]^{-1} \Psi+[I-\right.\right. \\
&\left.\left.\Psi M(0)]^{-1} \Psi R^{2}[I-N(0) M(\infty)]^{-1} N(0)\right] R\right]<1 \\
& \Leftrightarrow \bar{\lambda}\left[[ I + R [ I - \Psi M ( 0 ) ] ^ { - 1 } \Psi R ] \left[R[I-N(0) M(\infty)]^{-1}\right.\right. \\
&N(0) R-I]]<0 \\
& \Leftrightarrow \bar{\lambda}\left[[ I - R [ M ( 0 ) - \Psi ^ { - 1 } ] ^ { - 1 } R ] \left[R[I-N(0) M(\infty)]^{-1} N(0)\right.\right. \\
&R-I]<<0 \\
& \Leftrightarrow {\left[I-R\left[M(0)-\Psi^{-1}\right]^{-1} R\right]^{1 / 2}\left[R[I-N(0) M(\infty)]^{-1}\right.} \\
&N(0) R-I]\left[I-R\left[M(0)-\Psi \Psi^{-1}\right]^{-1} R\right]^{1 / 2}<0(\text { since } \\
&\left.I-R\left[M(0)-\Psi^{-1}\right]^{-1} R>0 \text { via Lemma } 21\right) \\
& \Leftrightarrow \bar{\lambda}\left[R[I-N(0) M(\infty)]^{-1} N(0) R\right]<1 \\
& \Leftrightarrow \bar{\lambda}\left[[I-N(0) M(\infty)]^{-1} N(0) R^{2}-I\right]<0 \\
& \Leftrightarrow \bar{\lambda}\left[[I-N(0) M(\infty)]^{-1}[N(0) M(0)-N(0) M(\infty)-I+\right. \\
&N(0) M(\infty)]<<0 \\
& \Leftrightarrow \bar{\lambda}\left[[I-N(0) M(\infty)]^{-1}(N(0) M(0)-I)\right]<0 . \\
&
\end{aligned}
$$

In Lemma 34, we state the equivalence between the stability conditions of Theorem 26 and Theorem 14 under the specialisation that $M(s)$ has no poles at the origin. 
Lemma 34: Let all the assumptions of Theorem 26 hold and furthermore suppose $M(s)$ has no poles at the origin. Then

$$
\begin{aligned}
& \bar{\lambda}\left[\operatorname { l i m } _ { s \rightarrow 0 } \left[[I-M(s) \Psi]^{-1}[M(s) N(\infty)-I][I-M(\infty)\right.\right. \\
& \left.\left.N(\infty)]^{-1}[I-M(\infty) \Psi]\right]\right]<0 \\
\Leftrightarrow & \bar{\lambda}\left[(M(0) N(\infty)-I)[I-M(\infty) N(\infty)]^{-1}\right]<0 .
\end{aligned}
$$

Proof: The proof is similar to that of Lemma 33 except that the second inequality in Theorem 26 is used instead of the third inequality in Theorem 24.

\section{Existence and Construction of a Negative Definite Matrix $\Psi$}

In Subsections V-B and V-C, we have derived powerful internal stability conditions that allow for the negative imaginary systems with free body dynamics. Some of the conditions in Theorems 24 and 26 and Corollary 31 are expressed in terms of a negative definite matrix $\Psi$ that is not part of the problem data. In the Corollary 31, since $M(\infty)=0$, then any choice of a negative definite $\Psi$ is fine. However, if the plant is not strictly proper, i.e. $M(\infty) \neq 0, \Psi<0$ also needs to satisfy $\bar{\lambda}[M(\infty) \Psi]<1$ as required by the assumptions of Theorems 24 and 26. We first demonstrate that such a $\Psi$ always exists, even when $M(\infty) \neq 0$, and give a simple constructive procedure to determine one such $\Psi$.

Since $M(\infty) \neq 0$ is a symmetric matrix, $M(\infty)$ can be factored into $M(\infty)=Q \Lambda Q^{T}$ where $Q$ and $\Lambda$ are orthogonal and diagonal matrices respectively. Now, choose $\Psi=Q \Lambda_{1}^{-1} Q^{T}$ where $\Lambda_{1}$ is a diagonal matrix with strictly negative numbers on its diagonal. It is clear that $\Psi<0$. Then, via 1) in Lemma 23, $\bar{\lambda}[M(\infty) \Psi]<1$ is equivalent to $M(\infty)-\Psi^{-1}>0$. Hence, the problem reduces to selecting a strictly negative definite and diagonal matrix $\Lambda_{1}$ which satisfies $\Lambda-\Lambda_{1}>0$. It is obvious that there always exists a matrix $\Lambda_{1}$ fulfilling $\Lambda_{1}<\Lambda$. Hence, it is trivial to construct a matrix $\Psi<0$ such that $\bar{\lambda}[M(\infty) \Psi]<1$.

It is trivial to observe that there is a large set of matrices $\Psi<0$ satisfying $\bar{\lambda}[M(\infty) \Psi]<1$. The statements in Theorems 24 and 26 and Corollary 31 indicate that any arbitrary $\Psi$ in this set could be selected. The following two theorems show that the conditions that depend on $\Psi$ in Theorems 24 and 26 and Corollary 31 are either fulfilled for all $\Psi<0$ satisfying $\bar{\lambda}[M(\infty) \Psi]<1$ or violated for all $\Psi<0$ satisfying $\bar{\lambda}[M(\infty) \Psi]<1$. Hence, choosing any arbitrary $\Psi<0$ satisfying $\bar{\lambda}[M(\infty) \Psi]<1$, for example via the constructive procedure described earlier, will be all that is required to use the powerful results in Theorems 24 and 26 and Corollary 31.

Theorem 35: Let $M(s)$ be a negative imaginary system and $N(s)$ be a strictly negative imaginary system. Define $\underline{X}=\{\Psi<0: \bar{\lambda}[M(\infty) \Psi]<1\}$. Then $\exists \Psi_{1} \in$ $\underline{X}: \bar{\lambda}\left[\lim _{s \rightarrow 0}\left[\left[I-\Psi_{1} M(\infty)\right][I-N(s) M(\infty)]^{-1}[N(s) M(s)-\right.\right.$ $\left.\left.I]\left[I-\Psi_{1} M(s)\right]^{-1}\right]\right]<0$ if and only if $\forall \Psi \in$ $\underline{X}, \bar{\lambda}\left[\lim _{s \rightarrow 0}\left[[I-\Psi M(\infty)][I-N(s) M(\infty)]^{-1}[N(s) M(s)-\right.\right.$ $\left.\left.I][I-\Psi M(s)]^{-1}\right]\right]<0$.

Proof:

$(\Leftarrow)$ Trivial.

$(\Rightarrow)$ Choose an arbitrary $\Psi \in \underline{X}$. Let $M_{1}(s)=M(s)[I-$ $\Psi M(s)]^{-1}, N_{1}(s)=N(s)-\Psi$, and $R=I-\left[M_{1}(0)-\right.$
$\left.M_{1}(\infty)\right]^{1 / 2}\left[I+\left(\Psi-\Psi_{1}\right) M_{1}(0)\right]^{-1}\left(\Psi-\Psi_{1}\right)\left[M_{1}(0)-\right.$ $\left.M_{1}(\infty)\right]^{1 / 2}$. See Lemma 8 for proof that $M_{1}(0)-$ $M_{1}(\infty) \geq 0$ after noting that $M_{1}(s)$ is negative imaginary without poles at the origin via Lemma 20. Then

$\bar{\lambda}\left[\lim _{s \rightarrow 0}\left[\left[I-\Psi_{1} M(\infty)\right][I-N(s) M(\infty)]^{-1}[N(s) M(s)-\right.\right.$ $\left.\left.I]\left[I-\Psi_{1} M(s)\right]^{-1}\right]\right]<0$

$\Leftrightarrow \bar{\lambda}\left[\lim _{s \rightarrow 0}\left[\left[I-\Psi_{1} M(\infty)\right][I-\Psi M(\infty)]^{-1}[I-\Psi M(\infty)][I-\right.\right.$ $N(s) M(\infty)]^{-1}[N(s) M(s)-I][I-\Psi M(s)]^{-1}[I-$ $\left.\left.\Psi M(s)]\left[I-\Psi_{1} M(s)\right]^{-1}\right]\right]<0$

$\Leftrightarrow \bar{\lambda}\left[\left[-I+\left[I-N_{1}(0) M_{1}(\infty)\right]^{-1} N_{1}(0)\left[M_{1}(0)-M_{1}(\infty)\right]\right]\right.$ $\left[I-\left[I+\left(\Psi-\Psi_{1}\right) M_{1}(0)\right]^{-1}\left(\Psi-\Psi_{1}\right)\left[M_{1}(0)-M_{1}(\infty)\right]\right.$ ]] $<0$

$\Leftrightarrow \bar{\lambda}\left[\left[-I+\left[M_{1}(0)-M_{1}(\infty)\right]^{1 / 2}\left[I-N_{1}(0) M_{1}(\infty)\right]^{-1}\right.\right.$ $\left.\left.N_{1}(0)\left[M_{1}(0)-M_{1}(\infty)\right]^{1 / 2}\right] R\right]<0$ (via Lemma 22)

$\Leftrightarrow \bar{\lambda}\left[-I+\left[M_{1}(0)-M_{1}(\infty)\right]^{1 / 2}\left[I-N_{1}(0) M_{1}(\infty)\right]^{-1}\right.$ $\left.N_{1}(0)\left[M_{1}(0)-M_{1}(\infty)\right]^{1 / 2}\right]<0$ (since $R>0$ via 6$)$ in Lemma 23)

$\Leftrightarrow \bar{\lambda}\left[\lim _{s \rightarrow 0}\left[[I-\Psi M(\infty)][I-N(s) M(\infty)]^{-1}[N(s) M(s)-\right.\right.$ $\left.\left.I][I-\Psi M(s)]^{-1}\right]\right]<0$.

The result then follows since $\Psi \in \underline{X}$ was arbitrary.

Necessity of Theorem 35 states that if there exists a $\Psi<0$ satisfying $\bar{\lambda}[M(\infty) \Psi]<1$ and the third condition in Theorem 24 (i.e. the condition involving the matrix $\Psi$ ), then this third condition of Theorem 24 is satisfied for all $\Psi<0$ satisfying $\bar{\lambda}[M(\infty) \Psi]<1$. The contrapositive statement of this same necessity of Theorem 35 states that if there exists a $\Psi<0$ satisfying $\bar{\lambda}[M(\infty) \Psi]<1$ that violates the third condition of Theorem 24, then this third condition of Theorem 24 is violated for all $\Psi<0$ satisfying $\bar{\lambda}[M(\infty) \Psi]<1$.

Theorem 36 is similar to Theorem 35, but tackles the second inequality of Theorem 26 (i.e. the one involving the matrix $\Psi$ ) instead of the third inequality of Theorem 24.

Theorem 36: Let $M(s)$ be a negative imaginary system and $N(s)$ be a strictly negative imaginary system. Define $\underline{X}=\{\Psi<0: \bar{\lambda}[M(\infty) \Psi]<1\}$. Then $\exists \Psi_{1} \in \underline{X}: \bar{\lambda}\left[\lim _{s \rightarrow 0}\left[\left[I-M(s) \Psi_{1}\right]^{-1}[M(s) N(\infty)-I][I-\right.\right.$ $\left.\left.M(\infty) N(\infty)]^{-1}\left[I-M(\infty) \Psi_{1}\right]\right]\right]<0$ if and only if $\forall \Psi \in \underline{X}, \bar{\lambda}\left[\lim _{s \rightarrow 0}\left[[I-M(s) \Psi]^{-1}[M(s) N(\infty)-I][I-\right.\right.$ $\left.\left.M(\infty) N(\infty)]^{-1}[I-M(\infty) \Psi]\right]\right]<0$.

Proof: Proof is omitted as it is similar to that of Theorem 35 .

Theorem 36 states that the second inequality of Theorem 26 is either satisfied for all $\Psi<0$ with $\bar{\lambda}[M(\infty) \Psi]<1$ or violated for all $\Psi<0$ with $\bar{\lambda}[M(\infty) \Psi]<1$. Hence, one only needs any arbitrary $\Psi<0$ with $\bar{\lambda}[M(\infty) \Psi]<1$ to use the result in Theorem 26.

\section{NumERICAL EXAMPLES}

Two numerical examples are given to illustrate the internal stability results developed in the previous sections.

\section{A. Without Poles at the Origin}

In this subsection, an example is given to illustrate the inapplicability or conservativeness of earlier results and demonstrate the main results developed in this paper. Let us consider 


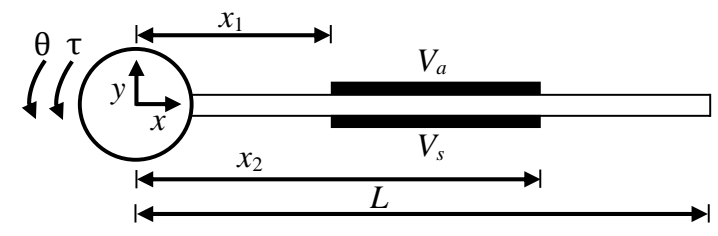

Fig. 2. Schematic diagram of a slewing beam equivalent to a robotic arm.

a positive feedback interconnection $[M(s), N(s)]$ as shown in Fig. 1. An undamped SISO plant $M(s)$ is given by $M(s)=\frac{1}{s^{2}+1}+2$ which is negative imaginary without poles at the origin according to [11, Definition 1]. A SISO controller $N(s)$ is given by $N(s)=\frac{1}{s+1}+2$ which is strictly negative imaginary according to Definition 2 . The internal stability of $[M(s), N(s)]$ cannot be determined via [11, Theorem 1], since $M(\infty) N(\infty)=4 \neq 0$. Also, [23, Corollary 4.3] cannot be used to check the internal stability of $[M(s), N(s)]$ since $\bar{\sigma}(M(0) N(0))=9 \nless 1$ and $\bar{\sigma}(M(\infty) N(\infty))=4 \nless 1$. Next, we attempt to use the IQC-based sufficient conditions of [23, Theorem 4.2]. We will demonstrate via this example that the sufficient conditions of [23, Theorem 4.2] are conservative. Let $\Pi_{0}=\Pi_{0}^{*}=\left[\begin{array}{ll}a & b \\ b & c\end{array}\right]$ and $\Pi_{\infty}=\Pi_{\infty}^{*}=\left[\begin{array}{ll}d & e \\ e & f\end{array}\right] \cdot[23$, Theorem 4.2] is only applicable if there exist scalars $a, b, c$, $d, e$, and $f$ such that the sufficient conditions in [23, Theorem 4.2] are satisfied for all $\tau \in[0,1]$. However, $\left[\begin{array}{c}N(0) \\ I\end{array}\right]^{*} \Pi_{0}$ $\left[\begin{array}{c}N(0) \\ I\end{array}\right]=9 a+6 b+c<0$ and $\left[\begin{array}{c}I \\ \tau M(0)\end{array}\right]^{*} \Pi_{0}\left[\begin{array}{c}I \\ \tau M(0)\end{array}\right]$ $=9 c \tau^{2}+6 b \tau+a \geq 0$ cannot be fulfilled simultaneously when $\tau=1 / 9$. Hence, [23, Theorem 4.2] cannot be used here.

By applying Theorem 17 in Section IV, we can easily conclude that $[M(s), N(s)]$ is internally stable since either condition 2) or 3) is satisfied, i.e. $M(\infty) N(\infty)=4>1$, $M(0) N(0)=9>1$ and $M(\infty) N(0)=6>1$. The robust stability conditions in this paper are easy to check, are necessary and sufficient, and only depend on the steady-state and instantaneous gains (i.e. the 0 and $\infty$ frequency gains) of the systems.

\section{B. With Poles at the Origin}

In this subsection, a physically motivated example is presented to demonstrate the application of the generalised feedback stability results derived in this paper. The example is directly taken from [12] for ease of comparison. Let us consider a flexible robotic arm, as shown in Fig. 2, which is driven by a motor mounted at one end and with two piezoelectric patches attached to either side of the arm which are used as an actuator and a sensor respectively. The control inputs of this flexible structure are the voltage $V_{a}$ applied to the actuator patch and the torque of the motor $\tau$, while the outputs of the system are the voltage $V_{s}$ generated by the sensor patch and the motor hub angle $\theta$. Using techniques detailed in [28], this flexible robotic arm can be modelled as an infinite dimensional transfer function: $G(s)=\frac{1}{D(s)}\left[\begin{array}{cc}N_{\tau, \theta}(s) & N_{V_{a}, \theta}(s) \\ N_{\tau, V_{s}}(s) & N_{V_{a}, V_{s}}(s)\end{array}\right]$, where
$N_{\tau, \theta}(s), N_{V_{a}, \theta}(s), N_{\tau, V_{s}}(s), N_{V_{a}, V_{s}}(s)$, and $D(s)$ are given in equations (26) - (28) in [28]. Through approximation approaches, we can represent the above model $G(s)$ as the finite dimensional model and for the sake of simplification we here only consider the finite dimensional model $M(s)$ with only the first resonant mode which can be expressed as $M(s)=\sum_{i=0}^{1}\left[\begin{array}{cc}\frac{a_{i}}{s^{2}+p_{i}^{2}} & \frac{b_{i}}{s^{2}+p_{i}^{2}} \\ \frac{c_{i}}{s^{2}+p_{i}^{2}} & \frac{d_{i}}{s^{2}+p_{i}^{2}}\end{array}\right]$, where $a_{0}=0.14, b_{0}=$ $c_{0}=d_{0}=p_{0}=0, a_{1}=3.0907, b_{1}=c_{1}=3.5573 \times$ $10^{-4}, d_{1}=2.35$, and $p_{1}=3.4$ [12]. This finite dimensional model $M(s)$ is a negative imaginary system since all poles of $M(s)$ are located in the closed left-half plane, $j[M(j \omega)-$ $\left.M(j \omega)^{*}\right]=0$ for all $\omega \in(0, \infty) \backslash 3.4$, the residue matrix $\lim _{s \rightarrow j 3.4}(s-j 3.4) j M(s)=\left[\begin{array}{cc}\frac{3.0907}{6.8} & \frac{3.5573 \times 10^{-4}}{6.8} \\ \frac{3.5573 \times 10^{-4}}{6.8} & \frac{2.35}{6.8}\end{array}\right]>0$ and $\lim _{s \rightarrow 0} s^{2} M(s)=\left[\begin{array}{cc}0.14 & 0 \\ 0 & 0\end{array}\right] \geq 0$ which imply the conditions 3) and 4) in Definition 1 are fulfilled. We then apply the strictly negative imaginary controller [3] $N(s)=(s I+$ $\Gamma \Phi)^{-1} \Gamma-\Delta$, where $\Gamma=\left[\begin{array}{ll}35 & 15 \\ 15 & 20\end{array}\right], \Phi=\left[\begin{array}{ll}0.745 & 0.521 \\ 0.521 & 1.021\end{array}\right]$ and $\Delta=\left[\begin{array}{cc}4.29 & 0 \\ 0 & 2.22\end{array}\right]$, as detailed in [12]. Note that since $M(s)$ is a negative imaginary system with two poles at the origin, the internal stability results introduced in [11] and [23] are not applicable. Hence, for ease of calculation, we directly set $\Psi=N(0)<0$ and use the generalised stability results presented in Subsection V-C. Using the stability condition in 2) of Corollary 31, we get $\bar{\lambda}\left[\lim _{s \rightarrow 0}\left[[N(s) M(s)-I][I-\Psi M(s)]^{-1}\right]\right]=$ $\bar{\lambda}\left[\lim _{s \rightarrow 0}\left[[N(0) M(s)-I][I-N(0) M(s)]^{-1}\right]\right]=-1<0$. Hence, feedback stability of $[M(s), N(s)]$ is guaranteed for the chosen controller via 2) in Corollary 31 without any complicated calculations. In contrast, the results in [12] require computation of several matrix factorisations and also a set of different inequalities need to be checked for different cases that depend on the positive or negative semidefiniteness of a specific matrix which is a result of such factorisations in order to determine whether the positive-feedback interconnection is internally stable or not.

\section{CONCLUSION}

We have removed restrictive assumptions from the robust stability analysis of positive feedback interconnections of negative imaginary systems, and generalised results for negative imaginary systems that may also have poles at the origin. New necessary and sufficient conditions have thus been derived that reduce to the earlier necessary and sufficient conditions when the assumptions of earlier literature are imposed. This demonstrates that the new results generalise the earlier work in the area. We also show that it is possible to simplify the new necessary and sufficient conditions by imposing alternative new succinct assumptions that were not known in prior literature. Because the proposed extended internal stability results rely on a negative definite matrix $\Psi$ with specific properties, it was also shown that such a matrix always exists. Moreover, a systematic procedure was given for constructing $\Psi$ to fulfil 
the required properties. We also prove that no search is needed for an appropriate $\Psi$ as the results are valid for any arbitrarily chosen $\Psi$. Two numerical examples are given that demonstrate the completeness of the derived results in this paper and illustrate the knowledge gap in the earlier literature.

\section{ACKNOWLEDGEMENT}

The authors would like to thank an anonymous reviewer for suggesting some useful SISO specialisations.

\section{REFERENCES}

[1] A. Lanzon and I. R. Petersen, "Stability robustness of a feedback interconnection of systems with negative imaginary frequency response," IEEE Transactions on Automatic Control, vol. 53, no. 4, pp. 1042-1046, May 2008.

[2] A. Ferrante, A. Lanzon, and L. Ntogramatzidis, "Foundations of not necessarily rational Negative Imaginary systems theory: Relations between classes of Negative Imaginary and Positive Real systems," IEEE Transactions on Automatic Control, vol. 61, no. 10, pp. 3052 3057, Oct. 2016.

[3] I. R. Petersen and A. Lanzon, "Feedback control of negative-imaginary systems," IEEE Control Systems Magazine, vol. 30, no. 5, pp. 54-72, Oct. 2010.

[4] M. A. Mabrok, A. G. Kallapur, I. R. Petersen, and A. Lanzon, "Spectral conditions for negative imaginary systems with applications to nano-positioning," IEEE/ASME Transactions on Mechatronics, vol. 19, no. 3, pp. 895-903, June 2014.

[5] B. Bhikkaji, S. O. R. Moheimani, and I. R. Petersen, "A negative imaginary approach to modeling and control of a collocated structure," IEEE/ASME Transactions on Mechatronics, vol. 17, no. 4, pp. 717-727, Aug. 2012.

[6] I. A. Mahmood, S. O. R. Moheimani, and B. Bhikkaji, "A new scanning method for fast atomic force microscopy," IEEE Transactions on Nanotechnology, vol. 10, no. 2, pp. 203-216, Mar. 2011.

[7] C. Cai and G. Hagen, "Stability analysis for a string of coupled stable subsystems with negative imaginary frequency response," IEEE Transactions on Automatic Control, vol. 55, no. 8, pp. 1958-1963, Aug. 2010.

[8] J. Wang, A. Lanzon, and I. R. Petersen, "Robust output feedback consensus for networked negative-imaginary systems," IEEE Transactions on Automatic Control, vol. 60, no. 9, pp. 2547-2552, Sept. 2015.

[9] _ - "Robust cooperative control of multiple heterogeneous negative-imaginary systems," Automatica, vol. 61, pp. 64-72, Nov. 2015.

[10] J. Xiong, I. R. Petersen, and A. Lanzon, "On lossless negative imaginary systems," Automatica, vol. 48, no. 6, pp. 1213-1217, June 2012.

[11] _ "A negative imaginary lemma and the stability of interconnections of linear negative imaginary systems," IEEE Transactions on Automatic Control, vol. 55, no. 10, pp. 2342-2347, Oct. 2010.
[12] M. A. Mabrok, A. G. Kallapur, I. R. Petersen, and A. Lanzon, "Generalizing negative imaginary systems theory to include free body dynamics: Control of highly resonant structures with free body motion," IEEE Transactions on Automatic Control, vol. 59, no. 10, pp. 2692 2707, Oct. 2014.

[13] M. R. Opmeer, "Infinite-dimensional negative imaginary systems," IEEE Transactions on Automatic Control, vol. 56, no. 12, pp. 2973-2976, Dec. 2011.

[14] A. van der Schaft, "Positive feedback interconnection of Hamiltonian systems," in Proceedings of the 50th IEEE Conference on Decision and Control and European Control Conference, Orlando, FL, USA, Dec. 2011, pp. 6510-6515.

[15] J. Xiong, A. Lanzon, and I. R. Petersen, "Negative imaginary lemmas for descriptor systems," IEEE Transactions on Automatic Control, vol. 61, no. 2, pp. 491-496, Feb. 2016.

[16] A. Ferrante and L. Ntogramatzidis, "Some new results in the theory of negative imaginary systems with symmetric transfer matrix function," Automatica, vol. 49, no. 7, pp. 2138-2144, July 2013.

[17] A. Ferrante, A. Lanzon, and L. Ntogramatzidis, "Discrete-time negative imaginary systems," Automatica, vol. 79, pp. 1-10, May 2017.

[18] J. Xiong, I. R. Petersen, and A. Lanzon, "Finite frequency negative imaginary systems," IEEE Transactions on Automatic Control, vol. 57, no. 11, pp. 2917-2922, Nov. 2012.

[19] S. Patra and A. Lanzon, "Stability analysis of interconnected systems with 'mixed' negative-imaginary and small-gain properties," IEEE Transactions on Automatic Control, vol. 56, no. 6, pp. 1395-1400, June 2011.

[20] Z. Song, A. Lanzon, S. Patra, and I. R. Petersen, "Towards controller synthesis for systems with negative imaginary frequency response," IEEE Transactions on Automatic Control, vol. 55, no. 6, pp. 1506-1511, June 2010.

[21] — , "Robust performance analysis for uncertain negative-imaginary systems," International Journal of Robust and Nonlinear Control, vol. 22, no. 3, pp. 262281, Feb. 2012.

[22] — , "A negative-imaginary lemma without minimality assumptions and robust state-feedback synthesis for uncertain negative-imaginary systems," Systems and Control Letters, vol. 61, no. 12, pp. 1269-1276, Dec. 2012.

[23] S. Z. Khong, I. R. Petersen, and A. Rantzer, "Robust feedback stability of negative imaginary systems: An integral quadratic constraint approach," in Proceedings of the 14th European Control Conference, Linz, Austria, July 2015.

[24] M. A. Mabrok, A. G. Kallapur, I. R. Petersen, and A. Lanzon, "A generalized negative imaginary lemma and Riccati-based static state-feedback negative imaginary synthesis," Systems and Control Letters, vol. 77, pp. 63-68, Mar. 2015.

[25] K. Zhou, J. C. Doyle, and K. Glover, Robust and Optimal Control. Upper Saddle River, NJ: Prentice-Hall, 1996. 
[26] M. Green and D. J. N. Limebeer, Linear robust control. Englewood Cliffs, NJ: Prentice Hall, 1995.

[27] R. W. Newcomb, Linear Multiport Synthesis. New York: McGraw-Hill, 1966.

[28] H. R. Pota and T. E. Alberts, "Multivariable transfer functions for a slewing piezoelectric laminate beam," Journal of Dynamic Systems, Measurement, and Control, vol. 117, no. 2, pp. 352-359, 1995.

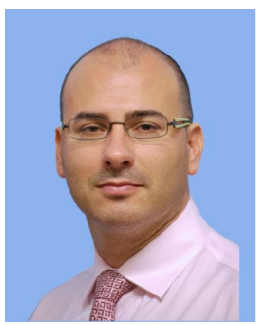

Alexander Lanzon received his Ph.D. degree in Control Engineering and his M.Phil. degree in Robot Control from the University of Cambridge in 2000 and 1997 respectively and received his B.Eng.(Hons). degree in Electrical and Electronic Engineering from the University of Malta in 1995. Alexander held academic positions at Georgia Institute of Technology and the Australian National University, and industrial posts at ST-Microelectronics (Malta) Ltd., Yaskawa Denki (Tokyo) Ltd. and National ICT Australia Ltd., before joining the University of Manchester in 2006 where he now holds the Chair in Control Engineering. Alexander is a Fellow of the Institute of Mathematics and its Applications, the Institute of Measurement and Control and the Institution of Engineering and Technology. He is also an Associate Editor of the IEEE Transactions on Automatic Control and has served as a Subject Editor of the International Journal of Robust and Nonlinear Control. His research interests include negative imaginary systems, robust control, fundamentals of feedback control theory, distributed/consensus control of multi-agent dynamical systems, and control applications for innovative aerial drones, robotics and mechatronic systems.

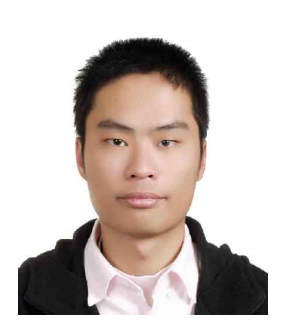

Hsueh-Ju Chen received the B.S. and M.S. degree in Mechanical Engineering from National Taiwan University of Science and Technology and National Taiwan University, Taiwan, in 2010 and 2012, respectively. $\mathrm{He}$ is a Ph.D. student currently studying in Control Systems at the University of Manchester, U.K.. His research interests are in negative imaginary systems, robust control, and suspension control. 